\title{
A Two-Sided Contracting Stefan Problem
}

\author{
Lincoln Chayes and Inwon C. Kim \\ January 8, 2008 \\ Department of Mathematics, UCLA, Los Angeles, CA 90059-1555 USA
}

\begin{abstract}
We study a novel two-sided Stefan problem - motivated by the study of certain 2D interfaces in which boundaries at both sides of the sample encroach into the bulk with rate equal to the boundary value of the gradient. Here the density is in $[0,1]$ and takes the two extreme values at the two free boundaries. It is noted that the problem is borderline ill-posed: densities in excess of unity liable to cause catastrophic behavior. We provide a general proof of existence and uniqueness for these systems under the condition that the initial data is in $[0,1]$ and with some mild conditions near the boundaries. Applications to 2D shapes are provided, in particular motion by weighted mean curvature for the relevant interfaces is established.
\end{abstract}

\section{Introduction}

A while ago, one of us - in a collaborative effort, [4] - investigated a number of physical problems leading to the study of certain one-dimensional Stefan-problems with multiple boundaries. We will begin with an informal (i.e. classical) description of the latter starting with the single boundary case. The problem is an archetype free boundary problem, here the boundary is denoted by $L(t)$. In the region $L \leq x$, there is a density $\rho(x, t)$ which is non-negative (and may be assumed identically zero for $x<L)$ and which satisfies the diffusion equation with unit constant

$$
\Delta \rho=\rho_{t}
$$

subject to Dirichlet conditions, with vanishing density, at $x=L$ :

$$
\rho(L(t), t) \equiv 0
$$

Finally, the bulk diffusion and the boundary motion are related by the Stefan condition

$$
\rho_{x}(L(t), t)=+\dot{L}(t)
$$

where the overdot denotes differentiation with respect to time $t$. The plus sign is emphasized: The more commonly studied Stefan problems have the opposite sign and have been well understood for several decades. Here, the problem is borderline ill-posed. In particular, for Equ.(1.3) as explicitly written - namely unit constant of proportionality relating the left and right hand sides - if the density is in excess of unity, catastrophic behavior may be exhibited. 
Physically, in a material setting, the problem corresponds to a supersaturated solution with the solute adhering to, and thence augmenting, the boundary. This description corresponds, more or less, to the stochastic particle system investigated in [4] which will be detailed in the subsequent paragraph. Alternatively, in a thermal context, the above system is a description of a supercooled liquid with a freezing boundary. Here the boundary is at, or close to, equilibrium below the freezing point, and will withdraw heat (or, more precisely, enthalpy) from liquid in its immediate vicinity. This serves to solidify the latter which in turn, again, augments the boundary.

The particulate version described above is readily modeled in the context of interacting particle systems. (Here, again, we are content with an informal description.) A favored choice for bulk behavior, which anyway will prove quite useful later, is the symmetric exclusion process. At time $t$, the sites $i \in \mathbb{Z}$ are occupied by a particle or are vacant, $\eta_{t}(i)=1$ or $\eta_{t}(i)=0$ respectively with the latter sometimes referred to as holes. At random times a particle is designated to move one space to the left or right with equal probability. The particle will do so provided that the site is not already occupied but, should the position be blocked, the particle simply retains its current position. (The reader is invited to the book [14] for a more complete description.) This is the behavior of the bulk which is envisioned to take place to the right of the boundary. The boundary, located at $b_{t}$ is a vacant site; to the left lies an array of "deactivated" particles and to the right, the bulk. When $\eta_{t}\left(b_{t}+1\right)=1$ and this particle jumps to $b_{t}$ then the boundary moves: $b_{t} \rightarrow b_{t}+1$ and the afore mentioned particle now becomes part of the static array.

There is an interesting connection between the exclusion/Stefan problem as described and dynamical $2 \mathrm{D}$ interfaces. In particular, the interface problem will motivate a second boundary to add to the system in Eqs.(1.1)-Equ.(1.3) which might otherwise appear peculiar. First let us envision a 2D interface as edges separating sites of the square lattice $\mathbb{Z}^{2}$. We may regard sites - and the unit squares surrounding them as plus on one side of the interface and minus on the other. (Or, somewhat more accurately, the interface is the boundary of the region of plus-squares.) The dynamics is described as follows: At random times, a plus square changes to minus or vice versa provided this change does not cause the overall length of interface to increase. It is seen that, at least in a formal sense, this is the zerotemperature limit of the standard Glauber dynamics [9] for Ising systems. In this work, we consider a single monotone interface - that is the interface is the graph of a non-increasing function with vertical segments attached. Then, it is claimed, that for an infinite interface, the above described Glauber dynamics is in one-to-one correspondence with the symmetric exclusion process with vertical segments playing the rôle of particles and horizontal segments that of holes. (This interpretation was, to our knowledge, introduced in [15]; the system that starts out as an "infinite corner" is discussed in [14] in Ch.VIII.)

Finite interfaces that are monotone necessarily imply that the interface runs into the boundary of the sample. Consider a droplet of plus-spins in the corner of an otherwise all minus Ising system which occupies the positive quadrant. The region outside the quadrant is taken to be empty and, needless to say, the interface is assumed to be monotone. Spin transitions which occur at the boundary reduce the length of $+/$ - interface (replacing a + /e segment with a -/e segment which are regarded as equally costly) and are therefore irreversible. Therefore, it is seen that action on the left of the particle system 
is exactly the discrete Stefan problem described above. On the right site, there is another moving boundary in the particle system - the situation is identical under particle/hole symmetry but, at least for the authors, considerably more difficult to visualize.

These considerations motivate us to consider the system, described classically as a triple $\langle\rho(x, t), L(t)$, $R(t)\rangle$ with $L_{0}=L(0) \leq L(t) \leq R(t) \leq R(0)=R_{0}$ and $\rho(x, 0)=\rho_{0}(x)$ satisfying the free boundary problem

$$
\left\{\begin{array}{l}
\rho_{t}-\Delta \rho=0 \text { in }\{(x, t) \mid L(t)<x<R(t), t \geq 0\} \\
\rho(L(t), t)=0, \quad \dot{L}(t)=\rho_{x}(L(t), t) \\
\rho(R(t), t)=1, \quad \dot{R}(t)=-\rho_{x}(R(t), t) .
\end{array}\right.
$$

Of course the particle system described does not immediately generate the above system in classical form. However, in [4] it was demonstrated that the weak form of the above system is indeed associated with the problem of a (monotone) droplet in the positive quadrant in the limit as the lattice spacing tends to zero. It is worth mentioning, albeit briefly, that the problem of non-monotone droplets can also be described by particle systems. These models, which acquire a mechanism for the spontaneous nucleation of Stefan boundaries, are currently under investigation by the authors.

Previous work on the two-sided system is essentially non-existent. The one-sided problem was investigated in [6] where the Neumann problem with certain restrictions was studied. The existence of a solution with a $C^{1}$ boundary was established along with uniqueness among all solutions of that class. In [4] it was first demonstrated that the particle system must produce a continuous boundary (at least $C^{1 / 2}$ ). Then a Dirichlet problem was investigated with (required) restrictions on the magnitude of the data and (overly severe) restrictions on the initial data. Existence and uniqueness among solutions with a continuous free boundary was established. Finally, the work [2] (and some references therein) should be mentioned where certain complimentary results have been established by different methods. So far, the only result for the two-sided problem is when there is complete symmetry under reflection about the midpoint and $\rho \rightarrow 1-\rho$ in the initial condition (corresponding to a droplet with symmetry under reflections through the line at $45^{\circ}$ eminating from the origin). This reduces the problem to a pair of decoupled matching Dirichlet problems with boundary density identically equal to $1 / 2$ on the line of symmetry.

The central purpose of this work is to rectify the paucity of results available for the two sided problem - and thereby correspondingly enhance the understanding of droplet dynamics in these (albeit still restricted) cases. Roughly speaking, existence and uniqueness for the general form of the weak equations is established which by an osmotic effect, so to speak, has provided some substantial improvements for the one sided cases. Complete statements are forthcoming in the next section. 


\section{Statement of Results}

We will start with a presentation of the weak formulation for the one and two sided problems. These weak formulations actually involves a pair of functions $a(x, t)$ and $\rho(x, t)$ with $\rho$ the usual (interior) density and $a$, which will be referred to as the enthalpy. In addition to the actual equations, we will state some "essential" (e) hypotheses and some technical (t) hypotheses concerning the pair $(a, \rho)$. While it may be the case that some of the e-hypotheses are stated in a form that is stronger than strictly necessary, these items are genuinely required in the sense that without something along these lines, our main theorem is false.

The pair of functions determine the moving boundary/boundaries - later to be denoted by $L(t)$ and $R(t)$. For the present, the live region is defined by $a=\rho$ (a.e.) while in the static region(s), $a \equiv 1$ and $\rho \equiv 0$ is the frozen region ostensibly to the left of $L$. Similarly, if applicable, $a \equiv 0$ and $\rho \equiv 1$ is a frozen region ostensibly to the right of $R$.

The single sided problem takes place on $\left[L_{0}, K\right] \times[0, T)$ where $L_{0}$ is the initial point of the Stefan boundary, $K$ is the fixed boundary and $T<t^{\star}$ where $t^{\star}>0$ depends on details of the boundary data to be described later. The Dirichlet data at $x=K$ is denoted by $\rho_{F}(t)$. For $x=L_{0}$, the boundary condition for all $t$ is that $a\left(L_{0}, t\right)$ is identically one and $\rho\left(L_{0}, t\right)$ is identically zero. The initial data in the region $\left[L_{0}, K\right]$ is that $a(x, 0)=\rho(x, 0)=\rho_{0}(x)$. The two sided problem is defined on $\left[L_{0}, R_{0}\right] \times[0, T]$ with $T$ smaller than some time also denoted by $t^{\star}$ which is also determined by initial conditions; the initial condition is also denoted by $\rho_{0}$. The left boundary condition is the same and, at the right boundary, we have $a\left(R_{0}, t\right) \equiv 0$ while $\rho\left(R_{0}, t\right) \equiv 1$.

The hypotheses read as follows:

\section{Hypotheses e-H.1.}

- In general, for the one-sided problem, $\rho(x, t) \geq 0$ for a.e. $(x, t)$. For the two-sided problem a similar requirement must be made on $(1-\rho)$ and thence, in these cases, $0 \leq \rho \leq 1$ for a.e. $(x, t)$.

- For both the one and two-sided cases, the initial density $\rho_{0}(x)$ is a measurable function satisfying $0 \leq \rho_{0}(x) \leq 1$ for a.e. $x$. For the one-sided problem the boundary density at $x=K$, denoted by $\rho_{F}(t)$ is a measurable function with $\rho_{F}(t) \leq 1$ for a.e. $t$.

- Whenever $\rho \neq a$ then, in the one-sided case, $a=1$ and $\rho=0$ while in the two sided case either the preceding or $a=0$ and $\rho=1$.

- For all $t$, the region $\{x \mid a(x, t)=\rho(x, t)\}$ is connected.

For the one-sided version, let $G(x, t)$ denote a smooth test function on $\left[L_{0}, K\right] \times[0, T)$ assumed, for simplicity, to be a constant at $x=K: G(K, t) \equiv g$. Then

$$
\begin{aligned}
\int_{L_{0}}^{K}[a(x, s)(G(x, s)-g) & \left.-\rho_{0}(x)(G(x, 0)-g)\right] d x= \\
& \int_{L_{0}}^{K} \int_{0}^{s}\left[a(x, t) G_{t}(x, t)+\rho(x, t) G_{x x}(x, t)\right] d t d x-\int_{0}^{s} \rho_{F}(t) G_{x}(K, t) d t
\end{aligned}
$$


For the two-sided problem the weak equation is given, for an arbitrary smooth $G(x, t)$, by

$$
\begin{aligned}
\int_{L_{0}}^{R_{0}}[a(x, s) G(x, s) & \left.-\rho_{0}(x) G(x, 0)\right] d x= \\
& \int_{L_{0}}^{R_{0}} \int_{0}^{s}\left[a(x, t) G_{t}(x, t)+\rho(x, t) G_{x x}(x, t)\right] d t d x-\int_{0}^{s} G_{x}\left(R_{0}, t\right) d t .
\end{aligned}
$$

The above constitute the essential hypotheses as will be detailed below. In addition we will insert some hypotheses of a more technical nature. It may be presumed that could be considerably softened or even removed with additional labour.

Hypotheses t-H.2.

○ There is a positive constant $c_{0}<1$ such that in a neighborhood of $L_{0}$, the function $\rho_{0}<c_{0}$. Similarly, in the two-sided cases, at $x=R_{0}, 1-\rho_{0}$ is bounded above by $1-c_{0}$.

- For a.e. $t$, the function $\rho(x, t) \in L^{p}(d x)$ for some $p>1$ with a norm that is uniform in $t$.

Definition 2.1 Let $\langle\rho(x, t), a(x, t)>$ be a solution of (2.1). Then we define the boundary $L(t)$ by

$$
L(t)=\sup \{x: a(y, t)=1 ; \rho(y, t)=0 \text { for } y<x\} .
$$

For solutions of (2.2) the right and left boundary $(L(t), R(t))$ are similarly defined.

Note that, by our connectivity hypothesis, $(L, R)=\{a=\rho\}$ with a corresponding statement for the one-sided case.

The main theorem reads:

Theorem 2.2 Consider the one-sided and two-sided Stefan problems that satisfy H1 and H2. Then each of these systems has a unique solution. Moreover, the solution is classical with a density satisfying $\rho(x, t) \in[0,1]$ and with well defined boundaries, e.g. $L(t), R(t)$ in the two sided problem, that are $C^{\infty}$.

Remark 1. The final technical condition is typical in the usual construction of solutions via weak limits. The hypotheses concerning the initial and boundary data are, perhaps, stronger than absolutely necessary but nevertheless something along these lines is required. Indeed, if $\rho$ is permitted to exceed the critical value of unity in too close a proximity to the moving boundary, the ill-posed nature of the problem will be revealed. Later, we will provide a simple demonstration of this effect. Similarly, $\rho_{0}$ not identically one in a neighborhood of $L_{0}$ is an absolute requirement; our stipulation about $c_{0}$ is obviously too strong but a considerable improvement over the conditions employed in [4].

It is noted that solutions of the weak equations always exist, in particular via weak limits of the particle systems corresponding to droplet problems that were studied in [4]. Moreover, all of the hypotheses are satisfied (or, if relevant, can be satisfied) by these systems. Regardless of the particle models, classical solutions as described in the statement of Theorem 2.2 above will be constructed. Thus, a seminal issue is uniqueness.

The first and last e-conditions, which may seem to the reader to be both strong and peculiar are, in fact strict requirements. Indeed, at a glance it might appear that these properties are derivable 
from the governing equation and the initial condition but this is not the case. Back in the context of a particle system, the Stefan boundaries are grown from a single site. Thus, in addition the the situation at the left, we might have e.g. at $t=0$, a "seed" at some $x \in\left(L_{0}, K\right)$ - i.e. at some site near $N x$ - which for $t>0$ will extend a boundary outward in both directions. The limiting evolution will, in fact, obey the weak equation Equ.(2.1) and, one may presume, lead to a smooth solution which is unique "for the circumstances". However, in the initial data, $\rho$ and $a$ will only differ at a single point which, of course, cannot be detected in a weak formulation. We remark that the above mentioned seeding condition will lead to interesting two-sided boundary problems that differ from the one studied in the present note. Moreover, as alluded above, there are other multi-sided Stefan problems that naturally emerge in the context of $2 \mathrm{D}$ droplet problems in which spontaneous nucleation events are featured. These problems are currently under investigation and will, hopefully, be the subject of future papers. A similar circumstance holds with regards to the positivity assumption. Foremost, we remark that positivity is typically assumed even in the "stable cases" where the boundaries recede from the gradient (for example see p. 84 in [8]). It is likely that the forthcoming is pertinent even to these situations. In any case, again in the context of a particle system, one can envision a dynamic in which vacant sites "split" into a particle/antiparticle pair which can later recombine. As far as bulk behavior is concerned, it is clear that the limiting behavior with and without the splitting mechanism is identical. At the Stefan boundary, two dynamics can be envisioned: (1) The antiparticles could "melt" the boundary. (2) The antiparticles could be forbidden to enter the boundary region. Both types of processes have counterparts in genuine chemical systems (not to mention the esoteric topic of "Bekenstein-Hawking radiation" [10]) and it would appear that in both cases some microscopic version will satisfy the discretized analog of the weak equation. These problems are also currently under investigation by the authors, (and will, hopefully, ... papers). Let us discuss, informally, the latter. Consider the case where $\rho_{0}$ and $\rho_{F}$ are identically zero so that without the splitting mechanism, nothing happens - manifestly a solution to the weak equation. Now let us allow splitting which we may envision as a rapid process. Away from the boundary, vacancies split and recombine quickly which produces a negligible overall effect. However when a vacancy near the boundary splits, the particle may increment the boundary leaving the antiparticle free to join the bulk. Since a buildup of antiparticles at the boundary will, directly or indirectly, inhibit future renditions of this event it is clear that antiparticles must diffuse away for further incrementing to occur. Away from the boundary, there is no net source of antiparticle; the gradient of antiparticle density is negative rendering the gradient of the "particle" density positive. And, as is not hard to envision, the growth rate of the boundary will be proportional to this gradient.

Therefore, the system described by Equ.(2.1) without the supplementary connectivity and positivity hypotheses is describing different problems physically which makes uniqueness completely hopeless mathematically. As we will show in the appendix, these assumptions (along with the "technicalities") allow the complete reduction to an essentially classical problem whereby uniqueness is established.

The 2D droplet corresponding to the two-sided Stefan problem is confined to the region $[0, W] \times$ 
$[0, H]$ with

$$
H=\int_{L_{0}}^{R_{0}} \rho_{0}(x) d x, \quad W=R_{0}-L_{0}-H=\int_{L_{0}}^{R_{0}}\left(1-\rho_{0}(x)\right) d x .
$$

Perhaps the best description of the shape is in parametric form; we denote the parameter by $q \in$ $[L(t), R(t)]$. Then

$$
Y(q, t)=H-\left(L-L_{0}\right)-\int_{L}^{q} \rho(x, t) d x
$$

with a similar formula ${ }^{1}$ for $X(q, t)$. From a straightforward computation, vindicated by the regularity result of Theorem 2.1, it follows that the dynamic evolution of the droplet is described by a weighted motion by mean curvature which is as follows:

Corollary 2.3 Consider the dynamical curve described parametrically by $X(q, t), Y(q, t)$ as in Equ.(2.4) and let $v(q, t)$ denote the inward normal velocity at the point $\langle X(q, t), Y(q, t)\rangle$. Then

$$
v=c \frac{\kappa}{\sigma^{2}(\hat{n})}
$$

where $\kappa=\kappa(q, t)$ is the curvature, $\hat{n}=\langle\cos \vartheta, \sin \vartheta\rangle$ is the normal vector to the curve, $c$ is a constant and $\sigma$ is the direction dependent surface energy given by the formula

$$
\sigma=|\cos \vartheta|+|\sin \vartheta|
$$

Proof. This is a standard exercise which follows from the parametric formula for the curvature. We note that similar results have been obtained for systems of this sort in [16], [12].

Remark 2. We remark that even with the present extensions, these results still restrict us to droplets in the corner of the magnetic/material sample. While it is tempting to state that our results cover fully immersed droplets with 4-fold symmetry, this is not quite the case: Indeed (unlike the $45^{\circ}$ symmetry exploited in [4]) a full treatment of non-monotone droplets requires a different and more sophisticated sort of particle system. Nevertheless, in these cases of symmetry, there is little doubt that the ultimate solution is exactly what is anticipated by these symmetry considerations.

\section{Proofs}

\subsection{Strategic Outline}

For the two boundary problem, the overall plan is not terribly dissimilar from the one-sided strategies employed earlier that are based on the construction of upper and lower sequences of monotone approximates; for the lower sequence, the $k^{\text {th }}$ pair of boundaries is inside the $k-1^{\text {st }}$ and vice versa for the upper sequence. However, there are several obstructions which must be overcome before a smooth-running procedure can be developed. First, there is no longer a fixed wall at which we can await the arrival of the boundary - we must carefully anticipate the time and place where the moving

\footnotetext{
${ }^{1}$ In [4] this formula was written down but, unfortunately, the constant term was accidently omitted.
} 
boundaries will collide. Second, while it is clear these upper and lower schemes ought to produce some sort of monotonicity, it is not immediately clear exactly what object ends up being monotone. Finally, as it turns out, in the case of the upper sequence it is slightly non-trivial getting the machinery started.

In order to better describe the scheme - as well as the crucial object of monotonicity - it will be necessary to introduce some notation. Let us consider a general system of the type under study, namely a Dirichlet problem in the subset of $\left[L_{0}, R_{0}\right] \times[0, T)$ determined by preprescribed boundaries $D(t) \leq E(t)$ with $\rho(D, t)=0$ and $\rho(E, t)=1$. Assuming, initially, that $D$ and $E$ are sufficiently smooth and then we define the following (integrated) fluxes:

$$
\Phi(D, E ; t)=\int_{0}^{t} \nabla \rho(D(s), s) d s
$$

and

$$
\Psi(D, E ; t)=\int_{0}^{t} \nabla \rho(E(s), s) d s .
$$

For $(x, t)$ such that $x \in[D(s), E(s)]$ for all $s<t$ there is also

$$
\Upsilon(D, E ; x, t)=\int_{0}^{t} \nabla \rho(x, s) d s .
$$

We remark that even in cases where the boundary is not so smooth - so that some interpretation of the right sides of Eqs.(3.1) and (3.2) might be required - the quantities $\Phi$ and $\Psi$ can usually be defined. Here, as it turns out, we are only interested in cases where the boundaries are monotone and continuous. Then, for any $x \in(D(t), E(t)), \Upsilon(x, t)$ is always well defined. In fact since $\rho$ is bounded via parabolic scaling, one can check that $\left|\Upsilon\left(x, s_{1}\right)-\Upsilon\left(x, s_{2}\right)\right| \leq O\left(\left|s_{1}-s_{2}\right|^{1 / 2}\right)$. Thus, if necessary, the boundary fluxes may be defined in terms of these objects e.g. $\Phi(t)=\lim _{s \rightarrow t} \Upsilon(D(t), s)+\int_{L_{0}}^{D(t)} \rho_{0}(y) d y$. However, in most of what follows, the "classical" definitions will suffice and this is what should be kept in mind for the forthcoming discussion.

The construction scheme is far easier for the case of the lower sequence and we will start with a fairly complete description. The first pair of boundaries $\left(\underline{L}_{0}, \underline{R}_{0}\right)$ is simply the pair $\left(L_{0}, R_{0}\right)$ itself. Then, $\left(\underline{L}_{1}, \underline{R}_{1}\right)$ is determined by the fluxes through the initial pair and similarly for later pairs. Explicitly

$$
\begin{aligned}
& \underline{L}_{k+1}(t)=L_{0}+\Phi\left(\underline{L}_{k}, \underline{R}_{k} ; t\right), \\
& \underline{R}_{k+1}(t)=R_{0}-\Psi\left(\underline{L}_{k}, \underline{R}_{k} ; t\right) .
\end{aligned}
$$

The upper sequence, $\left(\bar{L}_{k}, \bar{R}_{k}\right)$ is defined in the same way for short times but, it turns out, may have to be modified at later times for reasons that will shortly become clear. The logic of the scheme is based on the stipulation that if $\underline{L}_{k}$ lags behind a true Stefan boundary it stands to reason that the flux through $\underline{L}_{k}$ will exceed the distance this boundary has moved (namely $\underline{L}_{k}-L_{0}$ ) and hence the next boundary represents an improvement. A similar statement can be made about the upper sequence save for the unfortunate circumstance that here the fluxes could catch up with their own boundaries so a bit of modification is required. Moreover in this case it is not so obvious as to what boundaries should 
be used in the initial step of the construction; further discussion of this matter will be postponed till the next subsection.

Of course at this stage, it is by no means clear that the desired monotonicity, e.g. $\underline{L}_{k+1}(t) \geq \underline{L}_{k}(t)$ will even hold. The key item is a complete (pointwise) ordering of all the integrated fluxes for nested pairs of boundaries. I.e. if $D_{1} \leq D_{2}$ and $E_{1} \geq E_{2}$ then, for all relevant $(x, t)$ we have $\Upsilon\left(D_{1}, E_{1} ; x, t\right) \leq$ $\Upsilon\left(D_{2}, E_{2} ; x, t\right)$. This, with certain restrictions, will be the subject of Lemma 3.2 (which may be of some independent interest) and the necessary monotonicities follow immediately.

Finally, let us turn to the discussion of extinction times. From the prospective of the particle system, the location of the collision point is almost a triviality: Collision occurs when all the particles have migrated to the left and all the holes have migrated to the right. Thus, if we were to denote the collision point by $x^{\star}$ we would have

$$
x^{\star}=L_{0}+\int_{L_{0}}^{R_{0}} \rho_{0} d x=R_{0}-\int_{L_{0}}^{R_{0}}\left(1-\rho_{0}\right) d x ;
$$

however for the time being, we will define the quantity $x^{\star}$ by the above equation. Similarly, but not so obviously, there is a statistically conserved transport rate in the particle system (which has been discussed in [5], [4], [3]). This conservation law states that the total rate of transport - mass $\times$ distance - to the left is a constant - here unity. Thus, the two sided Stefan process stops when all the matter has been transported to the left. It is not hard to see that the total amount of transport necessary is independent of how the transport is achieved thus we may calculate the collision time by an arbitrary scheme. We introduce the function

$$
A(z)=\int_{L_{0}}^{z} \rho_{0}(x) d x
$$

which, among other mundane interpretations represents how far the left boundary has moved if all the material in $\left[L_{0}, z\right]$ is augmented to the boundary. If material is moved to the boundary on a "nearest first" priority, we arrive at

$$
d A=\frac{1}{z-A} d t=\rho_{0}(z) d z
$$

Equation (3.7) allows

$$
t^{\star}=\int_{L_{0}}^{R_{0}} d z[z-A(z)] \rho_{0}(z)
$$

which is our candidate for the collision time. The function $A$, especially with argument $t$ will play an important rôle in our later analysis and will be the featured item of Subsection $3.3^{2}$

\footnotetext{
${ }^{2}$ While the equations (3.6) and (3.7) can be gleaned directly from a continuum description, it is perhaps instructive to illustrate the concept at the level of the particle system. Here, simply put, the particle nearest the current location of the wall is transported at the allowed rate till it joins the wall. Now continuum quantities $A$, $t$, etc. are of order unity. Space, which is discrete, is related to continuum parameters by a factor of $N$ and time by a factor of $N^{2}$. Transport is also at rate unity (or $\rho_{F}$ ) for the lattice problem: one particle transported one unit to the left in one unit of simulation time. Thus, $N A$ marks the current location of the wall, $N z$ the initial location of the last particle that joined the wall which we may assume just happened at time $N^{2} t$. The next particle, located approximately at $N z+\rho_{0}^{-1}(z)$ takes time $N(z-A)+\rho^{-1}(z) \approx N(z-A)$ to move to the wall. This causes $N A \rightarrow N A+1$ and $N z \rightarrow N z+\rho_{0}^{-1}(z)-$ the latter approximate or statistical. Back on the unit (continuum) scale, this gives $\delta A=N^{-1}$ while $\delta z=N^{-1} \rho_{0}^{-1}(z)$ in time $\delta t=N^{-1}(z-A)$ which is the content of Eqs.(3.6) - (3.7).
} 
We end this subsection with a lemma which states that the heuristic predictions for the collisions are indeed correct:

Proposition 3.1 Suppose $\langle\rho(x, t), a(x, t)\rangle$ solve (2.2) described on $\left[L_{0}, R_{0}\right] \times[0, \mathbf{T})$ where $\mathbf{T}$ (the collision time) has the property

$$
\lim _{t \rightarrow \mathbf{T}} L(t)=\lim _{t \rightarrow \mathbf{T}} R(t)=: \mathbf{X}
$$

Then $\mathbf{X}=x^{\star}$ and $\mathbf{T}=t^{\star}$ with $x^{\star}$ and $t^{\star}$ given by Eqs.(3.5) and (3.8) respectively.

Proof. The continuum implementation of the conservation law amounts to the insertion of $G(x)=$ $R_{0}-x$ into Equ.(2.2) with the result

$$
\int_{L_{0}}^{R_{0}}\left(R_{0}-x\right) a(x, t) d x=t+\int_{L_{0}}^{R_{0}}\left(R_{0}-x\right) \rho_{0}(x) d x .
$$

Under the assumption of well defined boundaries, this gets us

$$
\begin{aligned}
\int_{L_{0}}^{R_{0}}\left(R_{0}-x\right) a(x, t) d x & =\int_{L}^{R}\left(R_{0}-x\right) \rho(x, t) d x+R_{0} L(t)-R_{0} L_{0}-\frac{1}{2} L(t)^{2}+\frac{1}{2} L_{0}^{2} \\
& =t+\int_{L_{0}}^{R_{0}}\left(R_{0}-x\right) \rho_{0}(x) d x .
\end{aligned}
$$

So, letting $t \rightarrow \mathbf{T}$, rearranging and using the definition of $x^{\star}$, we arrive at

$$
R_{0}\left(\mathbf{X}-x^{\star}\right)+\frac{1}{2} L_{0}^{2}-\frac{1}{2} \mathbf{X}^{2}=\mathbf{T}-\int_{L_{0}}^{R_{0}} x \rho_{0}(x) d x
$$

Next, we use the same (dual) idea to track "transport of holes": we insert $x-L_{0}$ as a test function against $(1-a)$ to obtain

$$
\begin{aligned}
\int_{L_{0}}^{R_{0}}\left(x-L_{0}\right)(1-a(x, t)) d x & =\int_{L}^{R}\left(x-L_{0}\right)(1-\rho(x, t)) d x+\frac{1}{2}\left(R_{0}^{2}-R^{2}\right)-L_{0}\left(R_{0}-R\right) \\
& =t+\int_{L_{0}}^{R_{0}}\left(x-L_{0}\right)\left(1-\rho_{0}(x)\right) d x .
\end{aligned}
$$

Performing the same sorts of steps that led to Eq.(3.12) we arrive at

$$
L_{0}\left(\mathbf{X}-x^{\star}\right)+\frac{1}{2} L_{0}^{2}-\frac{1}{2} \mathbf{X}^{2}=\mathbf{T}-\int_{L_{0}}^{R_{0}} x \rho_{0}(x) d x .
$$

Comparing the above with Equ.(3.12) we conclude that $\mathbf{X}=x^{\star}$ and, further, we have a formula for $\mathbf{T}$ which we claim implies $\mathbf{T}=t^{\star}$. Indeed, we may revamp Eq.(3.7) as $d A(z-A)=d t$ to arrive at

$$
t^{\star}=\int_{L_{0}}^{X}[z(A)-A(z)] d A=\int_{L_{0}}^{R_{0}} x \rho_{x} d x-\frac{1}{2}\left(\mathbf{X}^{2}-L_{0}^{2}\right) .
$$

This agrees with the formula for $\mathbf{T}$ from either Equ.(3.14) (or Equ.(3.12)) since it has already been agreed that $\mathbf{X}=x^{\star}$. 


\subsection{Flux and Transport Lemmas}

We now provide a proof of a key claim, a transport inequality, which reads as follows:

Lemma 3.2 Let $\left(D_{1}, E_{1}\right)$ and $\left(D_{2}, E_{2}\right)$ denote continuous monotone boundaries with $\rho_{1}$ and $\rho_{2}$ solving the heat equation in the appropriate domain as described prior to Equ.(3.1) and let $\Upsilon_{1}$ and $\Upsilon_{2}$ denote the associated quantities defined in Equ.(3.3). Suppose that the boundaries are ordered: $D_{1}(t) \leq D_{2}(t)$, $E_{1}(t) \geq E_{2}(t)$ with $D_{1}(0)=D_{2}(0)$ and $E_{1}(0)=E_{2}(0)$ and with initial densities in $[0,1]$ that are identical. Then, for all $(x, t)$ with $x \in\left(D_{2}(t), E_{2}(t)\right)$,

$$
\Upsilon_{1}(x, t) \leq \Upsilon_{2}(x, t)
$$

and further (as is anyway implied by Equ.(3.16)) $\Phi_{1} \leq \Phi_{2}$ and similarly for the $\Psi$ 's.

Remark 3. We remark that more generality is possible, e.g. we could have strict ordering in the initial conditions and $D_{1}(0)<D_{2}(0)$ (forcing system 1 to have vanishing initial density in $\left(D_{1}(0), D_{2}(0)\right.$ ) etc. Moreover, we could treat other boundary conditions instead of sources and sinks which are themselves ordered. However, this would complicate matters a bit. E.g. if the initial conditions are ordered, to run the forthcoming proof, we would need to introduce first and second class particles. Since this is not really needed for the present work we will stand with the more conservative claims.

Remark 4. We further remark that in this two-sided case, the situation is a bit more subtle than the single free boundary case. In the later, a straightforward argument using the maximum principle shows that the the densities are reverse ordered which orders the gradients at the fixed boundary. In the present case, if $D_{1}<D_{2}$ and $E_{1}>E_{2}$, ordering of the densities is obviously impossible. Naively it would seem that the gradients are ordered in $\left(D_{1}, E_{1}\right)$ but, as the following sort of example shows, there is in general no such ordering.

Example Let $\left(D_{2}, E_{2}\right) \subset\left(D_{1}, E_{1}\right)$ as in Lemma 3.2. and let $\rho_{1}$ and $\rho_{2}$ be the corresponding solutions of the heat equation as in Lemma 3.2. Let $E_{1}=E_{2} \equiv 1$. Let $D_{2}(t)=t$ and

$$
D_{1}(t)= \begin{cases}\frac{1}{2} t & \text { for } t \in[0,1 / 2], \\ 1 / 4+M t & \text { for } t \in[1 / 2,1 / 2+1 /(2 M)], \\ 3 / 4 & \text { for } t \in[1 / 2+1 /(2 M), 1] .\end{cases}
$$

Note that in the limit $M \rightarrow \infty, \rho_{1}$ is discontinuous at $\left(D_{1}(1 / 2), 1 / 2\right)$ and hence $\nabla \rho_{1}$ is a delta function at the point. In fact, if we change the boundary after $t=t_{0} \in[1 / 2+1 /(4 M), 1 / 2+1 /(2 M)]$ such that $D_{1}(t)=1 / 4+M t_{0}$ for $t \in\left[t_{0}, 1\right]$, then in the limit $M \rightarrow \infty$ one also obtains $\nabla \rho_{1}$ to be a delta function at $\left(D_{1}\left(t_{0}\right), t_{0}\right)$. Hence we conclude that if $M$ is large enough,

$$
\nabla \rho_{2}\left(D_{2}(t), t\right)<\nabla \rho_{1}\left(D_{1}(t), t\right) \text { for } t \in[1 / 2+1 /(4 M), 1 / 2+1 /(2 M)] .
$$

In our proof of Lemma 3.2 we resort to a discrete description of the circumstances using interacting particles. The crux will be proved in the context of a separate pre-lemma after which it is only a 
matter of some small formalities to prove Lemma 3.2 itself. The particles under consideration will obey symmetric exclusion on a time dependent subset of $\left\{D_{0}, D_{0}+1, \ldots, E_{0}\right\}$ with dynamic left and right boundaries $D(t)$ and $E(t)$ respectively. It is assumed that $D(0)=D_{0}$ and that $D(t)$ is non-decreasing with similar considerations for the $E$ 's. To implement the relevant dynamics, it is simply asserted that $\eta_{t}(D)$ is identically zero and $\eta_{t}(E)$ is always one and, provided the boundaries are static, everything runs according to the usual exclusion dynamics. Occasionally, the endpoints will move - in our case, deterministically at preprescribed times - in which case the best description of the dynamics is that in the new interval, the configuration is unchanged and at the new location of the boundary, $\eta$ takes on the appropriate value.

We wish to actualize the dynamics in such a way that we can keep track of all the particles. To this end, we envision two (ordered) stacks of particles, $\mathbb{S}_{D}$ and $\mathbb{S}_{E}$ located, respectively, at the position of the sink and source. Initially, $\mathbb{S}_{D}$ is empty while $\mathbb{S}_{E}$ starts (and stays) infinite and full. Particles are labeled by an integer $\alpha=1,2, \ldots$ starting at the leftmost particle and continuing indefinitely up the stack $\mathbb{S}_{E}$. The dynamics at the boundary is defined so that no particle is created or destroyed and the particles retain their initial order. In particular, for static boundaries, particles join the stack $\mathbb{S}_{D}$ and leave the stack $\mathbb{S}_{E}$ sequentially. Due to the motion of the boundaries, additional absorptions and emissions can take place. In particular, if the sink moves to a location currently occupied by a particle, the particle is absorbed - as a rule - and in our case gets place on top of the stack $\mathbb{S}_{D}$. If the source moves to a location that is currently occupied, since $\eta_{t}(E(t)) \equiv 1$ dynamically it is as though the particle were "still there". However, we do not destroy the previous occupant of the site, we allow this particle to get absorbed into $\mathbb{S}_{E}$ and take the position at the head of the stack (so it will be the first particle released in the next available emission). Finally we will associate random variables $\left(X_{\alpha}\right)$, one for each particle which tracks the position of the particle. In essence, $\left(X_{\alpha}\right)$ is the displacement of the particle to the left of the reference point $E_{0}$. Indeed, if the $\alpha^{\text {th }}$ particle is in the interior, that is $\{D(t)+1, \ldots, E(t)-1\}$ then $\left(X_{\alpha}\right)$ is simply $E_{0}$ minus the location of the particle. If the particle is in the right stack, then its position is identified with $E(t)$ and $X_{\alpha}(t)=E_{0}-E(t)$ and finally, if the particle has joined $\mathbb{S}_{D}$ then $X_{\alpha}$ is identically thereafter equal to $E_{0}-D_{0}$.

We claim that for a $\left(D_{1}, E_{1}\right),\left(D_{2}, E_{2}\right)$ nesting situation analogous to the one described in Lemma 3.2 , the arrays $\left(X_{\alpha}^{[1]}(t)\right)$ and $\left(X_{\alpha}^{[2]}(t)\right)$ are stochastically ordered. Explicitly

Lemma 3.3 Consider the setup described in the above paragraphs for two systems with the same initial configuration satisfying $D_{1}(t) \leq D_{2}(t), E_{1}(t) \geq E_{2}(t)$ (with $D_{1}(0) \leq D_{2}(0)=D_{0}$ and similarly for the $E$ 's). If the systems are coupled in a standard fashion wherein both systems are given the same marks delivered to the particles with instructions to jump forward or backward if practicable then for all $\alpha$ and for all $t$,

$$
X_{\alpha}^{[1]}(t) \leq X_{\alpha}^{[2]}(t)
$$

Proof. The overall situation is not terribly dissimilar to that of Theorem 2 in [3]. The desired statement is true at $t=0$, we need only check all possible finite time transitions to see that the ordering is maintained. We start with

Case 1: $X_{\alpha}^{[1]}(t)$ and $X_{\alpha}^{[2]}(t)$ correspond to particles both in the bulk. Suppose the particles get a message 
at time $t$. If already $X_{\alpha}^{[2]}(t)>X_{\alpha}^{[1]}(t)$, then, no matter what,

$$
X_{\alpha}^{[2]}\left(t^{+}\right) \geq X_{\alpha}^{[1]}\left(t^{+}\right) .
$$

Suppose then that they are equal and message says to move to the left. If the target in system-2 is unblocked, nothing can go wrong. But now suppose system-2 is blocked at this point. That would imply

$$
X_{\alpha-1}^{[2]}(t)=X_{\alpha}^{[2]}+1
$$

But $X_{\alpha-1}^{[1]}(t)$ satisfies

$$
X_{\alpha-1}^{[2]}(t) \geq X_{\alpha-1}^{[1]}(t)>X_{\alpha}^{[1]}(t)=X_{\alpha}^{[2]}(t)
$$

which implies that $X_{\alpha-1}^{[1]}(t)=X_{\alpha}^{[1]}(t)+1$ and system 1 is blocked as well.

Now suppose that the intended move is to the right; we only have to check the case when system 2 is unblocked. This means that $X_{\alpha+1}^{[2]}(t)<X_{\alpha}^{[2]}(t)-1$ but since $X_{j+1}^{[2]}(t) \geq X_{\alpha+1}^{[1]}(t)$ there is a corresponding vacancy available in the system 1 as well and the particles stay together.

Case 2: $X_{\alpha}^{[2]}$ corresponds to a particle at the right boundary. If the stacks are at equal location, then by hypothesis $X_{\alpha}^{[1]}$ also places its particle at the right boundary in an equal or worse relative position in the stack. Then there is nothing to prove. If $E_{1}(t)$ is to the right of $E_{2}(t)$, it may be the case that some particles have been released from $E_{1}(t)$ whose associates are still in $E_{2}(t)$. Let us consider such a particle, the $\alpha^{\text {th }}$ well inside the stack. We claim that the hypothesis at time $t$ implies that the associate is strictly behind $E_{2}(t)$. Indeed otherwise with the $\alpha-1^{\text {th }}$ particle of the second system still inside the stack and that of the first system in the bulk, we would necessarily violate $X_{\alpha-1}^{[2]} \geq X_{\alpha-1}^{[1]}$. So, for particles well inside the stack there is after all, nothing to prove. This leaves the subcase where $\alpha^{\text {th }}$ particle of the second system is the lead particle in the stack and the associate particle is in the same position but in the bulk. Right jumps are obviously not an issue and left jumps follow pretty much the same argument as in case 1.

Case 3: $X_{\alpha}^{[2]}$ corresponds to a particle at the left boundary. Note that in this case the particle is unblocked to the left where it is poised to increment its displacement as much as possible and to the right, it is more or less the same as the bulk situation.

Case 4: $X_{\alpha}^{[1]}$ corresponds to a particle at a boundary and $X_{\alpha}^{[2]}$ to a particle in the bulk. If $X_{\alpha}^{[1]}$ places the particle on the left boundary then the hypothesis will not allow the associate in the bulk. If $X_{\alpha}^{[1]}$ places the particle on the right boundary then there is nothing to prove.

Case 5: motion of stacks. Here there is not much to prove. If either of the system-2 boundaries move nothing is lost by absorption or motion. If the system- 1 boundaries move, nothing changes at the right - it must have been the case that $E_{2}$ was strictly greater than $E_{1}$ so, prior to the jump, all particles in the system- 1 stack were strictly behind their associates. If the system- 1 left boundary absorbs a particle then the hypothesis implies that the associate particle in system-2 had already been absorbed.

All cases have been covered.

At the discrete level, the exact analogs of the results stated for Lemma 3.2 are now obvious consequences. However, we must still get from the discrete to the continuous. While not terribly difficult, 
some caution is required since e.g. there is no existing technology for the direct proof of convergence for gradients. We proceed as follows:

Proof of Lemma 3.2 For the discrete system with any $(D(t), E(t))$ of the type described we let

$$
\rho_{N}(x, t)=\mathbb{E}\left(\eta_{N^{2} t}(N x)\right)
$$

be the value of our density on the grid sites and smoothed out - say to at least $C^{2}$ - in the regions in between. The relevant endpoint fluxes, from the perspective of the particle system are defined as

$$
S_{D ; N}=\mathbb{E}\left(\frac{1}{N}\left|\mathbb{S}_{D}\right|\left(N^{2} t\right)\right)
$$

where $\mathbb{E}$ denotes expectation in the particle system starting from a discrete approximation to $\rho_{0}$ and, $\left|\mathbb{S}_{D}\right|(t)$ denotes the size of the stack. A similar definition holds for for $S_{E ; N}$ (which we shall not be using) and, for future reference, the object inside the expectation will be denoted by $\mathbf{S}_{D ; N}(t)$. The first claim is that for all $t, \lim _{N \rightarrow \infty} S_{D ; N}$ exists; once this is established, it is not particularly difficult to identify the limit as $\Phi_{D}(t)$ (regardless of issues pertaining to the regularity of $\nabla \rho$ at the boundary). The starting point is the stipulation that $\rho_{N}$ or some subsequence thereof converges (e.g. weakly in $\left.L^{2}\right)$ to the appropriate solution of the heat equation which we denote by $\rho(x, t)$. Moreover, if $f(x, t)$ is a smooth test function and $F_{N}(t)=\int f(x, t) \rho_{N}(x, t) d x$ then

$$
F_{N}(t) \rightarrow F(t)
$$

where $F(t)=\int f(x, t) \rho(x, t) d x$ and again, e.g. the convergence is in $L^{2}$. Now for fixed $x$, let $f$ be a smooth monotone function whose derivative vanishes outside some $\varepsilon$-neighborhood of $x$ with $f \equiv 1$ to the left of the neighborhood and $f \equiv 0$ to the right. If $f_{j}$ denotes the discretized version, let us define the (random)

$$
\mathbb{K}_{f, N}=\sum_{j} f_{j} \eta_{t}(j)
$$

along with

$$
\mathbf{K}_{f, N}=\frac{1}{N} \mathbb{K}_{f, N}\left(N^{2} t\right) \quad \text { and } \quad K_{f, N}=\mathbb{E}\left(\mathbf{K}_{f, N}\right) .
$$

On the one hand, by weak convergence, and continuity of the known limit, $K_{f, N} \rightarrow \int \rho(y, t) f(y)$. We denote this limit by $K_{f}(x, t)$ and also, we have tacitly assumed that $t$ and $x$ are such that there is enough room for an $\varepsilon$-neighborhood of $x$ in $(D(t), E(t))$.

Meanwhile, letting $\Omega$ denote the evolution operator - semi-group generator + boundary terms for the particle system we have that (the martingale)

$$
\mathbf{K}_{f, N}(t)-\mathbf{K}_{f, N}(0)-\int_{0}^{t} \Omega \mathbf{K}_{f, N}(s) d s
$$

has zero expectation. It is noted that

$$
\Omega \mathbf{K}_{f, N}=-\Omega \mathbf{S}_{D ; N}(t)+\frac{1}{N} \sum_{j}\left[\Omega \eta_{t}(j)\right] f_{j}=-\Omega \mathbf{S}_{D ; N}(t)+\frac{1}{N} \sum_{j} \eta_{t}(j) \Delta f_{j}+\mathrm{o}(N)
$$


where the error term comes about from the discrete approximation to $f$. Taking expectations and rewriting a few terms we have

$$
K_{f, N}(t)-K_{f, N}(0)=-\int_{0}^{t} \mathbb{E}\left(\Omega \mathbf{S}_{\mathbf{D} ; \mathbf{N}}(s)\right) d s+\iint_{0}^{t} \rho_{N}(y, t) \Delta f(y) d y d s .
$$

Now of course $\int_{0}^{t} \mathbb{E}\left(\Omega \mathbf{S}_{\mathbf{D} ; \mathbf{N}}(s)\right) d s=S_{D ; N}(t)$; letting $N \rightarrow \infty$ and identifying all known weak limits, we now have the other hand for which

$$
K_{f}(t)-K_{f}(0)=-\lim _{N \rightarrow \infty} S_{D ; N}+\iint_{0}^{t} \rho(y, s) \Delta f(y) d y d s
$$

Thus the limit exists and, as is easily checked, it is independent of the location chosen for " $x$ " and the nature of the cutoff function. For smooth enough boundaries, Equ.(3.24) is already sufficient since

$$
K_{f}(t)-K_{f}(0)=-\int_{0}^{t} \nabla \rho(D(s), s) d s+\iint_{0}^{t} \rho(y, s) \Delta f(y) d y d s .
$$

In general we must proceed with a bit more caution. We first allow $f$ to approach a step function at $x$ and, in this limit, we can identify the second term as $\Upsilon(x, t)$. All the while the $K$-terms converge, unobtrusively to the contents of the intervals. The identification is complete by setting $x=D(t)$ and using $\Phi(t)=\lim _{s \uparrow t} \Upsilon(D(t), s)$.

An identical derivation allows a similar identification for the limit of $S_{E ; N}(t)$. As far as the boundary fluxes are concerned, the Lemma is proved since, on the basis of Lemma $3.3, \mathbb{S}_{D_{1}}(t) \leq \mathbb{S}_{D_{2}}(t)$ and similarly at the right. To settle the isssue for the internal integrated fluxes, we first note that $K_{f}(t)$ and $\int_{0}^{x} \rho(y, t) d y$ cannot differ by more than $2 \varepsilon$. Let us denote the functions with sharp cutoffs by $K$ 's which are unadorned by a subscript. Of course we will immediately adorn these with superscripts (along with the $K_{f}$ 's) to distinguish system-1 from system-2. We have for system-1

$$
\Upsilon_{1}(t)=K^{[1]}(t)-K^{[1]}(0)+\Phi_{1}(t)=K_{f}^{[1]}(t)-K_{f}^{[1]}(0)+\Phi_{1}(t)+\varepsilon_{1}
$$

with $\varepsilon_{1}<2 \varepsilon$ A similar equation holds for the second system. But back on the discrete system, it is clear that $K_{f}^{[1]}(t)+\Phi_{1}(t) \leq K_{f}^{[2]}(t)+\Phi_{2}(t)$ since in every realization of the coupling, at all times, $\mathbb{K}_{f}^{[1]}(t)+\mathbb{S}_{D_{1}}(t) \leq \mathbb{K}_{f}^{[2]}(t)+\mathbb{S}_{D_{2}}(t)$. The desired inequality has been established to within a few factors of $\varepsilon$ which we can remove at our leisure.

\subsection{Optimal Transport and Regularity}

Our next steps concern the auxiliary function $A(t)$, mentioned earlier in Eqs.(3.6) - (3.8). This function will be crucial for the establishment of regularity properties for solutions to Equ.(1.4) and in addition will provide the missing ingredient - the startup for the upper sequence. To recapitulate, in this system, there is a fixed rate of transport - perhaps more obvious at the level of the particle system - which is unity in the two-sided problem and $\rho_{F}(t)$ in the fixed right boundary case. The function $A(t)$ is designed to channel all the available transport into the construction of the boundary. In the two sided 
case, we will need two of these; for obvious reasons, the one on the left will be denoted by $A_{w}(t)$ and the one on the right by $A_{o}(t)$. I.e.

$$
d A_{w}(t)=\frac{1}{z_{w}(t)-A_{w}(t)} d t, \text { with } d A_{w}(t)=\rho_{0}\left(z_{w}(t)\right) d z(t) .
$$

On the right end, we do the same with $1-\rho$ instead of $\rho$ to construct $A_{o}(t)$ and $z_{o}(t)$ and, in the single boundary case, the equation is nearly the same with $d t$ replaced by $\rho_{F}(t) d t$. Note that since the density is in $[0,1]$ and strictly away from 1 in a neighborhood of $L_{0}, A_{w}$ is always to the left of $z_{w}$ and similarly $A_{o}$ is to the right of $z_{o}$.

Remark 5. It is instructive look at a few illustrations. First, it is noted that if $\rho_{0} \rightarrow \sigma$ as $x \rightarrow L_{0}$ then, for small times,

$$
A_{w}(t) \sim L_{0}+c_{a}\left(\frac{\sigma}{1-\sigma}\right)^{1 / 2} t^{1 / 2}
$$

for some constant $c_{a}$. In this case it turns out that $A$ behaves essentially the same as a Stefan boundary. Note the singular behavior as $\sigma \rightarrow 1$. However, if $\rho_{0}(x) \sim c_{1}\left(x-L_{0}\right)^{\beta}$ for $x$ close to $L_{0}$ then

$$
A_{w}(t) \sim L_{0}+c_{2} t^{\frac{\beta+1}{\beta+2}} .
$$

In the above, $c_{1}$ and $c_{2}$ are unimportant constants. Here it is noted (e.g. if $\beta=1$ ) that this is much faster than expected for a Stefan boundary.

For future reference, it is observed that in case $\rho_{0}$ tends to a small constant then the coefficient of $t^{1 / 2}$ is also small. Moreover, as is not hard to see in the discrete construction, a larger $\rho_{0}$ results in a larger $A(t)$. Since, for unrelated technical reasons we will later have to assume that $\rho_{0}$ is bounded by a small constant in a neighborhood of $L_{0}$, we may rest assured that for small times, $\left[A_{w}-L_{0}\right] t^{-1 / 2}$ is also bounded by a small constant.

Since our mid-range goals concern regularity of boundaries, as a first step, we provide regularity estimates for the $A(t)$ :

Lemma 3.4 Let $A_{o}, A_{w}$ denote the functions described in Equ.(3.28) and shortly thereafter and let $t^{\star}$ denote the extinction time as defined in Equ.(3.8). Then, under the assumption that $\rho_{0}$ is bounded strictly away from 1 on the left and 0 on the right, for any $0<t_{0}<t^{*}$ there is an $M_{0}<\infty$ such that

$$
\left|\dot{A}_{o}(t)\right|,\left|\dot{A}_{w}(t)\right| \leq M_{0} t^{-1 / 2} \text { for } 0 \leq t \leq t_{0} .
$$

Proof. We will demonstrate this on the left end (without subscripts); the argument on the right is identical after the usual transcriptions. It is first noted that if we examine as an independent entity the function $z(t)$, this function, while monotone can have discontinuities - in particular it will jump over regions where $\rho_{0}$ vanishes. Let us assume that this does not happen at $t=0$ for otherwise the argument is trivial and handled along the lines of the paragraph below. We use the Eqs.(3.28) to get

$$
d(z-A)=\frac{1-\rho_{0}}{\rho_{0}} \frac{1}{z-A} d t .
$$


By the boundedness of $\rho_{0}$, there is a $z_{0}$ such that $\rho_{0}(z) \leq c<1$ for $z<z_{0}$. Let $\tau_{0}$ be any positive time where $z \leq z_{0}$; it is noted that such a $\tau_{0}$ is possible by our working hypothesis. Then, for all $t<\tau_{0}$, we may bound

$$
\frac{1}{2} \frac{d}{d t}(z-A)^{2} \geq \frac{1-c}{c}
$$

and hence, still in this range of $t^{\prime}$ s, $(z-A) \geq \sqrt{c^{-1}(1-c)} t^{1 / 2}$. This can now be substituted directly into the first of Eqs.(3.28) to obtain the desired result for short times. Noting that with $\rho_{0}<1-$ at least for a while - during the initial stages of the evolution, a gap has opened up between $z$ and $A$ which, in light of the second of Eqs.(3.28) with $\rho_{0} \leq 1$ cannot decrease. Thus, if at time $\tau_{0}-$ which we now permit to include zero, we have $z\left(\tau_{0}\right)-A\left(t_{0}\right) \geq \Delta_{0}$ then, thereafter, $\dot{A} \leq \Delta_{0}^{-1}$. This can be patched together with the short time estimate to obtain the result in Equ.(3.31).

The following theorem is a one-dimensional version of the main theorem in [1].

Theorem 3.5 Suppose $\Sigma=\{(x, t): f(t) \leq x\}$, where $f$ is Lipschitz with Lipschitz norm $M_{0}$ and with $f(0)=0$. Suppose $\rho$ is a positive solution of the heat equation in $\Sigma \cap Q_{2}$, where $Q_{r}=B_{r}(0) \times(-r, r)$, continuously vanishing on $\partial \Sigma$. Then there exists a positive constant $M_{1}$, depending only on $M_{0}$ and the supremum of $u$ in $Q_{2}$, such that

$$
M_{1}^{-1} \frac{u(x, t)}{d_{x, t}} \leq|\nabla \rho|(x, t) \leq M_{1} \frac{u(x, t)}{d_{x, t}}
$$

in $Q_{1}$, where $d_{x, t}=x-f(t)$.

Proof. See the main theorem in [1].

Using Theorem 3.5, one can prove the following:

Lemma 3.6 If the boundary $(D, E)$ satisfies

$$
\dot{D}(t), \dot{E}(t) \leq M_{0} t^{-1 / 2} \text { for } t \leq t_{0}
$$

and if $\rho$ solves the heat equation as described prior to (3.1) with initial data $\rho_{0}<c_{0}=c_{0}\left(M_{0}\right)<1$ in $\left[L_{0}, L_{0}+2 \epsilon_{0}\right]$, then

$$
|\nabla \rho|(x, t) \leq M_{0} t^{-1 / 2} \text { for } t \leq \epsilon_{0}^{2}
$$

Proof. First note that, due to the Harnack inequality for heat equations,

$$
\rho\left(L_{0}+t^{1 / 2}, t\right) \leq C_{1}=C c_{0} \text { for } 0 \leq t \leq \epsilon_{0}^{2},
$$

where $C$ is a universal constant. We choose a small $c_{0}$ such that $C_{1} \leq \frac{M_{0}}{M_{1}}$. Then Theorem 3.5 yields the desired result.

Now we are now ready to prove the following: 
Lemma 3.7 Consider the system as described in Lemma 3.2 (see also the Equations (3.1) - (3.2)) with $(D(t), E(t))=\left(A_{w}(t), A_{o}(t)\right) ; 0 \leq t \leq t^{\star}$. Then there is a $t_{0}>0$ such that for all $t<t_{0}$

$$
\Phi_{A_{w}}(t)<A_{w}(t)-L_{0}, \quad \Psi_{A_{o}}(t)<R_{0}-A_{o}(t) .
$$

Proof. As usual, we will only check the left boundary using $A(t)=A_{w}(t)$ (also $z(t)=z_{w}(t)$ and we may as well set $L_{0}=0$. For emphasis, we will use $\Phi_{A}$ to denote the flux through $A$.

In case $\rho_{0}$ vanishes a.e. in a neighborhood of the boundary, it is seen that for short times, $\Phi_{A}$ is exponentially small (with rate $\propto-1 / t$ ) while $A$ grows linearly in $t$. Thus, we shall focus on the cases where $\int_{0}^{x} \rho_{0} d y>0$ for all $x>0$. Letting $t_{0}>0$ sufficiently small and defining $z_{0}=z\left(t_{0}\right)$ - assumed to satisfy $z_{0}<A_{o}(t)$ - we may write

$$
z_{0} A-\frac{1}{2} A^{2}=t+\int_{0}^{z}\left(z_{0}-x\right) \rho_{0}(x) d x
$$

which can be checked by differentiation and comparison of the result with Eqs.(3.28). On the other hand

$$
z_{0} \Phi_{A}-\int_{0}^{t} A \dot{\Phi}_{A} d s+\int_{A(t)}^{z_{0}}\left(z_{0}-x\right) \rho(x, t) d x=\int_{0}^{t} \rho\left(z_{0}, s\right) d s+\int_{0}^{z_{0}}\left(z_{0}-x\right) \rho_{0}(x) d x .
$$

Specializing both of the above equations to $t=t_{0}$ and neglecting the last term on the left of Equ.(3.35) we have

$$
z_{0} \Phi_{A}+\int_{0}^{t_{0}} A \dot{\Phi}_{A} d t+t_{0}-\int_{0}^{t_{0}} \rho\left(z_{0}, t\right) d t \leq z_{0} A-\frac{1}{2} A^{2}
$$

Next, it is claimed that if $t_{0}$ is sufficiently small then for some $\alpha>0$,

$$
\int_{0}^{t_{0}} \rho\left(z_{0}, t\right) d t<(1-\alpha) t_{0}
$$

This may be obtained by noting that $\rho(x, t)$ is, for short times, dominated by the density which has no boundary conditions, at $t=0$ agrees with $\rho_{0}$ in a small neighborhood of $x=0$ - vanishing for $x<0-$ and is slightly larger than one outside the neighborhood. Then, using the fact that for $x$ close to zero, $\rho_{0} \leq c<1$, the result follows. Moreover, if $\rho_{0}$ is sufficiently small in a neighborhood of the boundary, then $\alpha$ is close to one. Thus, after an integration by parts, we have an $\alpha \approx 1$ such that for small times,

$$
z_{0} \Phi_{A}-A \Phi_{A}+\int \Phi_{A} \dot{A} d t+\alpha t \leq z_{0} A-\frac{1}{2} A^{2}
$$

i.e.

$$
\Phi_{A}\left(z_{0}-A\right)+\alpha t \leq A\left(z_{0}-A\right)+\frac{1}{2} A^{2} .
$$

However (c.f. the sentence at the end of Remark 5) the small value of the initial $\rho_{0}$ near the boundary which provided us with the $\alpha$ close to one also bounds $A^{2}$ by a small constant times $t$. Since $z_{0}-A$ is positive, the result follows. 
Lemma 3.8 Consider a solution to Equ.(1.4) with left and right boundaries $(L(t), R(t))$. Then, starting from the same initial density, the boundaries $\left(A_{w}(t), A_{o}(t)\right)$ satisfy $A_{w} \geq L$ and $A_{o} \leq R$ for all times up to the extintion time.

Proof. We shall only prove $L \leq A\left(=A_{w}\right)$ and we make the convenient assumption that $L_{0}=0$. Note that for any $t_{0}>0$ and any $b_{0}$ with $R_{0} \geq b_{0} \geq L\left(t_{0}\right)$,

$$
b_{0} L\left(t_{0}\right)-\frac{1}{2} L^{2}\left(t_{0}\right)+\int_{0}^{b_{0}}\left(b_{0}-x\right) \rho\left(x, t_{0}\right)=\int_{0}^{t_{0}} \rho\left(b_{0}, s\right) d s+\int_{0}^{b_{0}}\left(b_{0}-x\right) \rho_{0}(x) d x .
$$

It is asserted that Equ.(3.40) is valid even if $b_{0}>R(t)$, provided we are willing to interpret $\rho(x, t)$ as identically one once $x$ is past $R(t)$. On the other hand, recapitulating Equ.(3.35)

$$
b_{0} A\left(t_{0}\right)-\frac{1}{2} A^{2}\left(t_{0}\right)+\int_{z\left(t_{0}\right)}^{b_{0}}\left(b_{0}-x\right) \rho_{0}(x) d x=t_{0}+\int_{0}^{b_{0}}\left(b_{0}-x\right) \rho_{0}(x) d x .
$$

If we suppose that $z\left(t_{0}\right) \geq L\left(t_{0}\right)$ then we may set $b_{0}=z\left(t_{0}\right)$ and we quickly learn that $b_{0} A-\frac{1}{2} A^{2} \geq$ $b_{0} L-\frac{1}{2} L^{2}$ which is the desired conclusion. But if we assume that $z\left(t_{0}\right) \leq L\left(t_{0}\right)$ we set $b_{0}=L\left(t_{0}\right)$ and arrive at

$$
L A-\frac{1}{2} A^{2}+\int_{z}^{L}(L-x) \rho_{0} d x \geq \frac{1}{2} L^{2} .
$$

Since $\rho_{0} \leq 1$ this would imply, after a small bit of algebra, that $L A-\frac{1}{2} A^{2} \geq L z-\frac{1}{2} z^{2}$ necessitating $z \leq A$ (or some other absurdity) and the desired result is established.

We now state (without proof) a standard Lemma which is in fact, a special case of Lemma 5 in [1].

Lemma 3.9 Let $f(t)$ satisfy $\left|f^{\prime}(t)\right| \leq M r^{-1 / 2}$ satisfying $f(0)=0$ and let $\Sigma_{f}=\{(x, t) \mid x \geq f(t)\}$. Let $V_{r}=\left[-M r^{1 / 2},+M r^{1 / 2}\right] \times[-r,+r]$ and let $\rho(x, t)$ denote a positive solution of the heat equation in $\Sigma \cap V_{r}$ vanishing on the boundary $\{x=f(t)\}$ with $\rho\left(M r^{1 / 2}, 0\right)=1$. Then there exist positive constants $0<\epsilon, \delta<1$, depending only on $M$ and the supremum of $\rho$ in $V_{r}$ such that

$$
\rho+\rho^{1+\epsilon} \text { is convex and } \rho-\rho^{1-\epsilon} \text { is concave in } V_{\delta r} \text {. }
$$

Using Lemma 3.9, one can prove the following short time estimate:

Lemma 3.10 Consider the system $(\rho(x, t), D(t), E(t))$ described prior to Equ.(3.1) with initial data $\rho_{0}(x)$ satisfying $\rho_{0}<c_{0}<1$ (with $c_{0}$ sufficiently small) in $\left[D(0), D(0)+\epsilon_{0}\right]$ and a similar consideration on the right. Suppose that the D-boundary satisfies

$$
\dot{D}(t) \leq M_{0} t^{-1 / 2} \text { for } t \leq t_{0}
$$

and similarly, for the E-boundary

$$
|\dot{E}|(t) \leq M_{0} t^{-1 / 2} \text { for } t \leq t_{0},
$$

with $t_{0}=\left(\frac{\epsilon_{0}}{2 M_{0}}\right)^{2}$, where $\epsilon_{0}$ is a small constant depending on $M_{0}$. Then for all $t<t_{0}$,

$$
|\nabla \rho|(D(t), t) \leq M_{0} t^{-1 / 2}
$$

and similarly for the E-boundary. 
Proof. We will only consider the boundary at $x=D(t)$, since the other half follows from a parallel argument. First suppose $\rho_{0}=c_{0}$ in $\left[D(0), D(0)+\epsilon_{0}\right]$ where $c_{0}=c_{0}\left(M_{0}\right)$ is to be described later.

For fixed time $t>0$, we would like to apply Lemma 3.9 to the parabolic cubes of scale $t / 2$ centered at $D(t)$ which we denote by $\Sigma_{0}$ :

$$
\Sigma_{0}=\left\{(x, \tau):|x-D(t)| \leq M_{0} \sqrt{t / 2}, \tau \in[t / 2, t]\right\} .
$$

For this purpose, first we need to check that the maximum of $\rho$ in $\Sigma_{0}$ is comparable to $\rho(D(t)+\sqrt{t} / 2, t)$.

When there is no right boundary $E$ present, one can apply the interior Harnack inequality of FabesGarofalo-Salsa [7] to check that $\rho$ is relatively homogeneous in the region $\Sigma$ which is similar to the above but now centered about $D(t)+M_{0} \sqrt{t}$. Since our right boundary $E$ is a distance of order unity away and $\rho=1$ (which is not "large") on $E$, for small $t$ the effect of $E$ on $\Sigma$ is negligible. Hence our $\rho$ is homogenous in $\Sigma$ if $t$ is sufficiently small.

Hence it remains to check whether $\rho$ stays smaller near the boundary $D$, that is in the region $\Sigma_{0}-\Sigma$ than in $\Sigma$. To see this, note that $\rho_{2} \leq \rho \leq \rho_{1}$, where $\rho_{1}$ solves the heat equation with $D=D(0)$ and with initial data $\rho_{0}$, and $\rho_{2}$ solves the heat equation with $D=D(t)$, with initial data $\rho_{0}$ restricted to $[D(t), E(0)]$. By looking at explicit formulae using Green's functions, one can check that the value of $\rho_{1}$ in $\Sigma_{0}-\Sigma$ is (with a constant multiple) smaller than that of $\rho_{2}$ in $\Sigma$. Hence one concludes that

$$
\max _{\Sigma_{0}} \rho \leq C \min _{\Sigma_{0}} \rho
$$

where $C$ depends on $M_{0}$.

Now we are able to apply Lemma 3.9 , to conclude that there exists some $\delta$ depending on $M_{0}, \epsilon_{0}$ such that,

$$
\left(\rho+\rho^{1+\epsilon}\right)(x, t) \leq \rho\left(D(t)+\delta t^{1 / 2}, t\right) \frac{(x-D(t))}{\delta t^{1 / 2}} \text { for } D(t) \leq x \leq D(t)+\delta t^{1 / 2}
$$

if $t$ is sufficiently small.

Now let us choose $c_{0} \leq \frac{1}{2} M_{0} \delta$. By comparing with exact solutions in $\mathbb{R} \times[0, t]$ with initial data $\rho_{0}$ and using the fact that $\rho_{0}<c_{0}$ in $\left[0, \epsilon_{0}\right]$ and $\rho_{0}<1$, we obtain that

$$
\rho\left(D(t)+\delta t^{1 / 2}, t\right) \leq 2 c_{0},
$$

if $t$ is sufficiently small depending on $M_{0}$. It follows that, for this range of $t$,

$$
\rho(x, t) \leq \frac{M_{0}(x-D(t))}{t^{1 / 2}} \text { for } D(t) \leq x \leq D(t)+\delta t^{1 / 2} .
$$

Therefore $\nabla \rho(D(t), t) \leq M_{0} t^{-1 / 2}$.

In general if $\rho_{0} \leq c_{0}$ on $\left[D(0), D(0)+\epsilon_{0}\right]$, by maximum principle $\nabla \rho(D(t), t) \leq \nabla \tilde{\rho}(D(t), t)$, where $\tilde{\rho}$ solves the heat equation in $(D, E)$ with same lateral boundary data as $\rho$ and initial data $\tilde{\rho}_{0}=c_{0}$ on $\left[D(0), D(0)+\epsilon_{0}\right]$. Hence the worst possible case has been treated above. 
The following lemma is a direct consequence of Lemma 3.4 and Lemma 3.10 and provides uniform $C^{1}$ bounds for the boundaries in the lower and upper sequences.

Lemma 3.11 Consider the setup $\rho, D, E$ described prior to Equ.(3.1) with $\epsilon_{0}, M_{0}$ and $t_{0}, c_{0}$ as described in the preceding lemmas. Suppose $D(t) \leq A(t)$ for $t \in[0, T]$ with $T<t^{*}$ with $t^{\star}$ the extinction time. Then for all $t \in[0, T]$ there exists a constant $W=W\left(c_{0}, \epsilon_{0}, t_{0}, M_{0}\right)$ such that if $\dot{D}(t) \leq F_{w}(t)$ with

(i) $F_{w}(t)=M_{0} t^{-1 / 2}$ for $t \in\left[0, t_{0}\right]$

(ii) $F_{w}(t)=\frac{W}{x^{*}-A_{w}(t)}$ for $t \in\left[t_{0}, T\right]$

where $x^{*}$ denote extinction point, then $\dot{\Phi}_{D} \leq F_{w}$ for all $t \in[0, T]$. Similar considerations hold at the right.

Proof. The statement for $0 \leq t \leq t_{0}$ holds due to Lemma 3.10.

Note that at $t=t_{0}$, due to the fact that $\dot{D}(t), \dot{A}(t) \leq M t_{0}^{-1 / 2}$ is uniformly bounded for $t_{0} / 2 \leq t \leq t_{0}$, $\rho$ has a linear growth near the left boundary, i.e.,

$$
\rho\left(x+D\left(t_{0}\right), t_{0}\right), \rho_{A}\left(x+A\left(t_{0}\right), t_{0}\right) \leq C x,
$$

where $C=C\left(t_{0}, M_{0}, c_{0}\right)$.

Also note that, due to Lemma 3.4, for $t \in\left[t_{0}, t^{*}\right], A(t)$ is differentiable with

$$
\dot{A}(t) \leq \frac{W}{x^{*}-A(t)}
$$

for $t \in\left[t_{0}, t^{*}\right]$ with $W=M_{0} t_{0}^{-1 / 2}$, and

$$
\dot{A}(t)=\frac{1}{z(t)-A(t)} \geq \frac{\epsilon}{x^{*}-A(t)}
$$

for $t_{0} \leq t \leq T<t^{*}$ with $\epsilon=\epsilon(T)>0, \epsilon \rightarrow 0^{+}$as $T \rightarrow t^{*}$. The last inequality holds because (i) $A(t)$ is strictly increasing in time, and therefore $x^{*}-A(t)>0$ for any $t<t^{*}$, (ii) $z(t)-A(t)=$ $\int_{0}^{z(t)}\left(1-\rho_{0}(y)\right) d y>0$ for all $t \in\left[0, t^{*}\right]$, and (iii) $z(t)$ and $A(t)$ are continuous. Due to (3.43),(3.44) and (3.45), one can apply Lemma A.2 in [CS] with our $A(t)$ with initial time $t=t_{0}$ to yield the conclusion for $t_{0} \leq t \leq T$.

\subsection{Proof of Main Results}

Proof of Theorem 2.2

\section{Construction of minimal and maximal solutions}

As described earlier, we construct upper and lower sequences of fixed boundary problems where the flux through the current boundary determines - partially or fully - the next boundary. For the 
lower sequence, we have $\left(\underline{L}_{0}, \underline{R}_{0}\right) \equiv\left(L_{0}, R_{0}\right)$ and $\left(\underline{L}_{k+1}, \underline{R}_{k+1}\right)=\left(L_{0}+\Phi_{k}, R_{0}-\Psi_{k}\right)$ where, in this context, $\Phi_{k}=\Phi_{k}(t)=\Phi\left(\underline{L}_{k}, \underline{R}_{k} ; t\right)$ etc. Of course once $k \geq 1$ we must terminate the process e.g. when the fixed boundaries collide, but we need not pay too much attention to such matters. Obviously $\left(\underline{L}_{1}, \underline{R}_{1}\right) \subset\left(\underline{L}_{0}, \underline{R}_{0}\right)$ - meaning $\underline{L}_{0}(t) \leq \underline{L}_{1}(t)$ and $\underline{R}_{0}(t) \geq \underline{R}_{1}(t)$ - and using Lemma 3.2, it is clear that $\left(\underline{L}_{k}, \underline{R}_{k}\right) \subset\left(\underline{L}_{k+1}, \underline{R}_{k+1}\right)$. We let $(\underline{L}, \underline{R})$ denote the limiting boundaries. Our first assertion is that these are sensible limits, in particular $\left(A_{w}, A_{o}\right) \subset(\underline{L}, \underline{R})$. Indeed, for general left boundary $D(t)$, assumed to satisfy $D<z$ we have, by specializing Equ.(3.41) to $b_{0}=z$,

$$
\int_{D}^{z}(z-x) \rho_{D}+z \Phi_{D}-\int_{0}^{t} D(s) \dot{\Phi}_{D}(s) d s=\int_{0}^{t} \rho_{D}(z(t), s) d s+\int_{0}^{z}(z-x) \rho_{0} d x \leq z A-\frac{1}{2} A^{2}
$$

where we have temporarily set $L_{0}=0$. Thus, after some manipulations,

$$
z \Phi_{D}-\frac{1}{2} \Phi_{D}^{2}+\frac{1}{2}\left(\Phi_{D}-D\right)^{2}+\int \dot{D}\left(\Phi_{D}-D\right) \leq z A-\frac{1}{2} A^{2} .
$$

It is thus evident that in any system where $\Phi_{D} \geq D$ (and $D<z$ ) the flux through $D$ does not exceed $A$; an easy version of Lemma 3.7 .

For present purposes, the initial boundary is obviously less than $A$, allowing us to conclude that $\Phi_{L_{0}} \leq A$ i.e. $\underline{L}_{1}<A$ and hence less than $z$ and we may quickly conclude that $\underline{L}_{k} \leq A \forall k$. Similar arguments take place at the right which vindicates the "first assertion".

Thus, the boundaries indeed converge to reasonable limits (which are below their $A$ counterparts). A simple argument shows that if $(\rho, L, R)$ is any Stefan system then $L \geq \underline{L}$ and $R \leq \underline{R}$. Indeed, as we demonstrate in the appendix, any solution to Equ.(2.2) or Equ.(2.1) has monotone and continuous boundaries. Supposing $(L(t), R(t))$ are the boundaries of one such solution. If it is known that $L \geq L_{k}$ and $R \leq R_{k}$ then according to Lemma $3.2, \Phi_{L} \geq \Phi_{\underline{L}_{k}}$; the first term equals $L$ by the Stefan condition and the second term equals $\underline{L}_{k+1}$ by definition. Similarly at the right boundary.

Next, the internal fluxes $\Upsilon_{k}(x, t)$ converge, by the monotonicity provided in Lemma 3.2, to a definitive limiting $\Upsilon(x, t)$. Further, since the densities are bounded we may extract a limit e.g. weakly in $L^{2}$. It is claimed that by standard arguments, the limit, $\rho$ satisfies the diffusion equation in the interior and, by slight elaboration of the standard arguments, the $\underline{\Upsilon}$ is the appropriate flux for the limiting density.

We perform a similar campaign for the upper sequence. Here $\left(\bar{L}_{0}, \bar{R}_{0}\right)$ is simply $\left(A_{w}, A_{o}\right)$. While austensibly $\bar{L}_{k+1}$ is given by the flux though $\bar{L}_{k}$, we want to keep the new boundaries outside of the old, i.e. $\left(\bar{L}_{k}, \bar{R}_{k}\right) \subset\left(\bar{L}_{k+1}, \bar{R}_{k+1}\right)$. By Lemmas 3.11 and 3.7, this is guaranteed for short times. But it may be the case that the flux catches up with the boundary. Thus we define

$$
\bar{L}_{k+1}=\min \left\{\bar{L}_{k}, L_{0}+\bar{\Phi}_{k}\right\}
$$

(where $\bar{\Phi}_{k}$ is notation for $\Phi\left(\bar{L}_{k}, \bar{R}_{k}\right)$ ) and similarly on the right. From Lemma 3.7 it is the case that $\bar{L}_{1}<\bar{L}_{0}$ for short times - and similarly on the right - and thereafter we apply Lemma 3.2 to demonstrate that the boundaries are genuinely receding. The comparison arguments can be applied to the upper sequence but, of course, this time, the flux inequalities tell us that these boundaries are contained in any Stefan boundaries. 
We thus have a second system $(\bar{\rho}, \bar{L}, \bar{R})$ and our next task will be to show that both of these are genuine solutions to the Stefan problem. Note that already, these systems satisfy a certain vague Stefan condition namely that the total flux through the boundary equals the displacement of the boundary itself. However this does not immediately imply the full system in Equ.(2.2) (or Equ.(2.1) in the single boundary case) without some additional regularity. Indeed specializing to $G(x, t)=\left(R_{0}-x\right)$ a Stephan system has

$$
\int_{L}^{R}\left(R_{0}-x\right) \rho(x, t) d x+(L-R) R_{0}-\frac{1}{2} L^{2}+\frac{1}{2} R^{2}=t+\int_{L_{0}}^{R_{0}}\left(R_{0}-x\right) \rho_{0}(x) d x
$$

whereas in our case, the (desired) $\frac{1}{2} L^{2}$ type term is replaced by a $\int_{0}^{t} \dot{\Phi}(s) L(s) d s$ term and similarly with the $R^{2}$ term. However, once the $\Phi$ 's, $\Psi$ 's etc. are outside the integrals, we are "safe" since our fluxes indeed converge to our boundary displacements. So, in the end, it is sufficient to show

$$
\lim _{k \rightarrow \infty} \int_{0}^{t}\left(L_{k}-\Phi_{k}\right) \dot{L}_{k} d s=0
$$

It is noted that for both the upper and lower sequences, we are in prime position to apply Lemma 3.11. First we reiterate that each " $L_{k}$ " is less than $A_{w}$ and similarly on the right. By fiat this is true for the upper sequence and as was argued in Eqs.(3.46) and (3.47) this is true for the lower sequence. As for the rest, by the nature of the lemma and the nature of the sequence, we need only verify the initial step and then subsequent steps hold automatically. Thus, $\dot{L}_{k}$ is uniformly bounded except for some controllable behavior at the origin. Equ.(3.50) now is easily established by first cutting out a neighborhood of the origin, where the explicit $t^{-1 / 2}$ bounds demonstrate a negligible contribution, and in the remainder employing dominated convergence.

Thus, both the upper and lower system are actually complete solutions to the Stefan problem; next we will establish regularity. Finally if we can show that they coincide we are done since according to the discussion above, these two solutions trap any other solution between them.

\section{II.Regularity}

By our hypothesis, $\rho_{0} \leq c_{0}$ in $\left[L_{0}, L_{0}+\epsilon_{0}\right]$ for some $\epsilon_{0}>0$. Due to Lemma 3.10 and Lemma 3.11, for $\tau_{0} \leq t \leq T<t^{*}$

$$
\left|\dot{\bar{L}}_{k}\right|,\left|\dot{\bar{R}}_{k}\right|,\left|\underline{\dot{L}}_{k}\right|,\left|\underline{\dot{R}}_{k}\right| \leq W \max \left(\frac{1}{x^{*}-A(t)}, t^{-1 / 2}\right)
$$

for some constant $W>0$ depending on $c_{0}, \epsilon_{0}$ and $T$. This yields uniform Lipschitz bound for both Stefan boundaries $(\bar{L}, \bar{R})$ and $(\underline{L}, \underline{R})$. To obtain further regularity, first note that if the boundary is Lipschitz in time, then $\nabla \rho$ is continuous in time. Hence the boundaries are in fact $C^{1}$. Now the hodograph methods yield that the $C^{1}$-free boundary is in fact $C^{\infty}$. This is done for obstacle problems by Isakov [11] and by Kinderlehrer-Nirenberg [13]. Since the boundary is $C^{1}, u(x, t)=\int_{t}^{t^{*}} \rho(x, s) d s$ solves the parabolic obstacle problem: 


$$
\left\{\begin{array}{l}
u_{t}-\Delta u=-\chi_{\{u(\cdot, t)>0\}}+\chi_{\left\{\rho_{0}>0\right\}} \\
u=|\nabla u|=0 \text { on } x=L(t), R(t) \\
u(x, 0)=0 .
\end{array}\right.
$$

(Necessary details can be acquired from the derivation in Di Benedetto-Friedman [2].)

\section{Uniqueness}

In the single sided case, uniqueness follows, presumably, from the argument in [6] and certainly from the derivation surrounding Equations (A.17)-(A.18) in [4]. For the two boundary case, a slightly different tack must be taken. Indeed we will require various arguments reminiscent of Lemma 3.3. In principle the particle system provides a great deal of additional information which may prove useful in other contexts. However, some further efforts will be required to convert these into continuum inequalities, so we will be content with a direct particle system argument for the present time. The cornerstone of the derivation is the two conservation laws, namely

$$
\int_{L_{0}}^{L} d x+\int_{L}^{R} \rho(x, t) d x=\mathrm{const} ; \int_{L_{0}}^{L}\left(R_{0}-x\right) d x+\int_{L}^{R}\left(R_{0}-x\right) \rho(x, t) d x=t+\text { const. }
$$

We shall show that the above implies that, e.g. $\underline{L}<\bar{L}$ and $\underline{R} \leq \bar{R}$ for Stefan systems is impossible. First, let $K$ satisfy

$$
\underline{L}+\int_{\underline{L}}^{K} \underline{\rho}(x, t) d x=\bar{L}
$$

or, perhaps more conveniently,

$$
\int_{K}^{\underline{R}} \underline{\rho} d x=\int_{\bar{L}}^{\bar{R}} \bar{\rho} d x
$$

Let us consider a discretization of these systems using the coupled exclusion dynamics featured in Lemma 3.2. It is emphasized that this is not a discrete Stefan system with a microscopic moving boundary of the type described earlier: Here we have sources and sinks that move deterministically in such a way that as $N \rightarrow \infty$, the rescaled total fluxes corresponds to the rescaled boundary displacements. In particular, the particle number itself can fluctuate so a little care must be taken.

For the two systems, we use the notation $\underline{X}_{\alpha}$ and $\bar{X}_{\alpha}$ to denote the displacement of the $\alpha^{\text {th }}$ particles from the rightmost edge (in microscopic units). Further, we use $\overline{\mathbb{L}}$ etc. to denote the microscopic location of the boundary in the upper system. The number of active particles in the upper system will serve as our reference; $\overline{\mathbb{N}}=|\overline{\mathcal{A}}|$ where

$$
\overline{\mathcal{A}}=\left\{\alpha \mid \overline{\mathbb{L}}<N R_{0}-\bar{X}_{\alpha}<\overline{\mathbb{R}}\right\}
$$

Recalling from the discussion prior to Lemma 3.3 the definition of the stack $\mathbb{S}_{\bar{L}}$, we have

$$
\mathbb{E}\left[\frac{1}{N}\left(\mathbb{S}_{\bar{L}}+\overline{\mathbb{N}}\right)\right] \rightarrow \int_{L_{0}}^{R_{0}} \rho_{0} d x \quad \text { and } \quad \mathbb{E}\left[\frac{1}{N}\left(\mathbb{S}_{\underline{L}}+\underline{\mathbb{N}}\right)\right] \rightarrow \int_{L_{0}}^{R_{0}} \rho_{0} d x
$$


Now before averaging, each particle in the upper system is ahead or equal with of its counterpart in the lower system. In particular, since $\underline{\mathbb{L}}<\overline{\mathbb{L}}$, no counterpart of a bulk particle in the upper system is in the lower stack. We thus have

$$
\sum_{i: \mathbb{\mathbb { L }}<i<\overline{\mathbb{R}}}\left[N R_{0}-i\right] \eta_{t}(i)=\sum_{\alpha \in \overline{\mathcal{A}}} \bar{X}_{\alpha} \geq \sum_{\alpha \in \overline{\mathcal{A}}} \underline{X}_{\alpha}
$$

where the inequality is the direct result of Lemma 3.3. On the other hand, we claim that the final term is approximately the transport contribution from $N K$ to $N \underline{R}$ in the lower system:

$$
\sum_{\alpha \in \overline{\mathcal{A}}} \underline{X}_{\alpha}=\sum_{i: N K<i<\underline{\mathbb{R}}}\left[N R_{0}-i\right] \eta_{t}(i)+\mathscr{E}_{N}
$$

with $\lim _{N \rightarrow \infty} N^{-1} E\left(\mathscr{E}_{N}\right)=0$. Indeed, the contributions to $\mathscr{E}_{N}$ come from the fraction of particles in the right stack and from particles which are on the "wrong side" of $N K$; both of these represent an asymptotically vanishing fraction of the average by convergence to the limiting diffusion equation. E.g. due to Eqs.(3.54) only a vanishing (average) fraction of the particles in the lower system corresponding to active particles in the upper system could be in the right stack. As an upshot, letting $N \rightarrow \infty$, we learn that

$$
\int_{K}^{\underline{R}}\left(R_{0}-x\right) \underline{\rho}(x, t) d t \leq \int_{\bar{L}}^{\bar{R}}\left(R_{0}-x\right) \bar{\rho}(x, t) d t .
$$

Now let us consider the remaining effective "enthalpy" in the two systems. By this it is meant the density defined to be unity to the left of the left boundaries and $\rho$ in the bulk. The upper system is concentrated on the interval $\left[L_{0}, \bar{L}\right]$ while the lower system is spread out over $\left[L_{0}, K\right]$ and is less than unity on $(\underline{L}, K)$; both systems have the same mass namely $\bar{L}-L_{0}$. It then follows, from elementary considerations, that

$$
\int_{L_{0}}^{\underline{L}}\left(R_{0}-x\right) d x+\int_{\underline{L}}^{K}\left(R_{0}-x\right) \underline{\rho} d x<\int_{L_{0}}^{\bar{L}}\left(R_{0}-x\right) d x .
$$

Hence the sums of the left sides of Eqs.(3.57) - (3.58) cannot equal the sums of the right sides whilst both are supposed to equal $t+\int_{L_{0}}^{R_{0}}\left(R_{0}-x\right) \rho_{0} d x$ by the transport conservation law.

\section{Appendix}

In this appendix, we establish some basic properties, namely monotonicity and continuity of the boundaries, for weak solutions of the Stefan problem as exemplified in Eqs.(2.1) and (2.2). It is noted that weak solutions always exist e.g. the construction in the proof of the main theorem and, in the present context more importantly, from the discrete version in the particle system. However to get from weak to strong - and uniqueness, two inputs are required namely monotonicity and continuity of the boundaries. In sharp contrast to [4], where these properties were extracted directly from the particle system, here we provide an $a b$ initio derivation (thus ruling out the intangible possibility that there are weak solutions exist which cannot be accessed by the particle system). Finally we will show that this system 
is genuinely unstable if it is allowed that $\rho>1$ in the vicinity of the boundary. In all cases we will, for simplicity work with the one-sided problem; parallel proof apply for the two-sided cases.

Our starting point (which is automatic for the two-sided case) is the natural bound on the density.

Proposition A.1. Consider the weak systems described under Hypotheses e-H.1 and t-H.2. Then, for a.e $(x, t), \rho(x, t) \leq 1$.

Proof. Recall that the boundary is defined by

$$
L(t)=\sup \{x \mid a(y, t) \equiv 1 ; \rho(y, t) \equiv 0 \text { for } y<x\} .
$$

Using $G(x, t)=G(x)=K-x$ and the fact that $\rho_{0}$ is, in the integral sense, strictly less than one and that $\rho_{F}(t)$ is bounded, it is clear that $L(t)$ stays bounded away from $K$ for a while. Thus there is an interior to the set where $\rho=a$ and, therein, classical diffusion is obeyed. Further, if $\rho$ is to get larger than the prescribed boundary values - which are always less or equal to one - such behavior must emanate from the boundary of the region where $\rho=a$.

Let us therefore entertain the possibility that at some time $t_{0}$, there is a $c>0$ such that on $\left(L\left(t_{0}\right), L\left(t_{0}\right)+c\right)$, the density exceeds one. We shall assume that this excess takes a weak form, partially because this is a form that easily propagates. Thus we shall say that $\rho$ weakly exceeds one if for any positive convex function $\alpha(x)$, which is positive at $L\left(t_{0}\right)$ and vanishes, along with all of its derivatives somewhere in $\left(L\left(t_{0}\right), L\left(t_{0}\right)+c\right)$, we find

$$
\int_{L\left(t_{0}\right)}^{L\left(t_{0}\right)+c} \rho \alpha d x \geq \int_{L\left(t_{0}\right)}^{L\left(t_{0}\right)+c} \rho d x .
$$

The upshot of this proposition is that should the above happen, the boundary immediately gets driven to $-\infty$. For simplicity we shall assume that $K=1$ that $L_{0}=0$ and we will use $C$ to denote $L\left(t_{0}\right)+c$. As for the up and coming, we will envision, informally, that $c$ is small; we note, for future reference that the weak equations between times $s$ and $t$ with $s>0$ read

$$
\begin{aligned}
& \int_{0}^{1}[a(x, t) G(x, t)-a(x, s) G(x, s)] d x \\
&=\int_{0}^{1} \int_{s}^{t}\left[a(x, t) \frac{\partial G}{\partial t}+\rho(x, \tau) \frac{\partial^{2} G}{\partial x^{2}} d x d \tau-\int_{s}^{t} \rho_{F}(\tau) \frac{\partial G}{\partial x}(1, \tau) d \tau .\right.
\end{aligned}
$$

The first consequence is that at times after $t_{0}$, the boundary indeed ceases to go forward. I.e. we claim that for all $t_{1}>t_{0}, L\left(t_{1}\right) \leq L\left(t_{0}\right)$. Supposing, on the contrary that $L\left(t_{1}\right)>L\left(t_{0}\right)$ and let $\tilde{L}$ denote the minimum of $L\left(t_{0}\right)+c$ and $L\left(t_{1}\right)$. Then, for a function $\alpha$ of the type described before Equ.(3.60) vanishing at $\tilde{L}$ Equ.(3.61) reads

$$
\int_{0}^{\tilde{L}} \alpha d x-\int_{0}^{L_{0}} \alpha d x-\int_{L_{0}}^{\tilde{L}} \rho\left(x, t_{0}\right) d x=\int_{t_{0}}^{t_{1}} \int_{0}^{\tilde{L}} \rho(x, t) \alpha^{\prime \prime} d x .
$$

The right hand side is positive (by the convexity and the positivity assumption) while the left side is negative if $\tilde{L}>L\left(t_{0}\right)$. Moreover, recapitulating the above argument we have that

$$
\int_{L\left(t_{1}\right)}^{C} \rho\left(x, t_{1}\right) \alpha(x) d x \geq \int_{L\left(t_{1}\right)}^{L\left(t_{0}\right)} \alpha(x) d x+\int_{L\left(t_{0}\right)}^{C} \rho\left(x, t_{0}\right) d x
$$


i.e. at all times later than $t_{0}$ the density (weakly) exceeds one in an interval at least as large as $c$ to the right of the boundary. Furthermore, we now have that at times later than $t_{0}$ the region to the right of $L\left(t_{0}\right)$ is essentially classical. We use this to show that for any $t>t_{0}$

$$
\int_{L(t)}^{C} \rho(x, t) \geq \frac{1}{2} c .
$$

Indeed, we employ the transport function with boundary data at $x=C$, i.e. $G(x)=C-x$ and note that throughout $[0, C], a\left(x, t_{0}\right)$ is never less than one. We arrive at

$$
\int_{L(t)}^{C}[C-x] \rho(x, t) d x-\frac{1}{2}(C-L(t))^{2} \geq \int_{t_{0}}^{t} \rho(C, t) d t \geq 0 .
$$

On the other hand, $\int_{L}^{C}[C-x] \rho d x \leq[C-L] \int_{L}^{C} \rho d x$ and $[C-L]$ is at least as large as $c$ which vindicates the claim in the above unlabeled display. Now, let us denote for $t=t_{0}+b$ the backwards displacement $\Delta(b)$ defined by $L\left(t_{0}+b\right)=L\left(t_{0}\right)-\Delta(b)$. Our next claim is that for $c$ small and $b$ also small but considerably larger than $c$, there is a $\theta<1$ such that

$$
\Delta(b) \geq m[b]^{\theta} .
$$

Here $m>0$ depends on the $L^{p}$ norm of $\rho$ as described in the second of the t-H.2 hypotheses. We resort to a quadratic function that vanishes at (or "just before") $x=C$-i.e. $G=(C-x)^{2}$ and by our previous argument

$$
\int_{L\left(t_{0}\right)-\Delta}^{L\left(t_{0}\right)+c}[\rho-1] G d x \geq 2 \int_{t_{0}}^{t_{0}+b} d t \int_{L(t)}^{C} \rho(x, t) d x .
$$

As a consequence of the preceding claim we can replace the right hand side by $b c$. As for the left hand side, we neglect the one, pull out a $(\Delta+c)^{2}$ and estimate

$$
\int_{L\left(t_{0}\right)-\Delta}^{L\left(t_{0}\right)+c} \rho\left(x, t_{0}+b\right) d x \leq M[\Delta+c]^{1 / q}
$$

where $M$ is the $L^{p}$ norm of $\rho$ and $q$ is the complimentary index. Thus far we have

$$
(c+\Delta)^{2+\frac{1}{q}} \geq m^{\prime} c b
$$

Now let us take $b=c^{\kappa}$ with $\kappa>q /(q+1)$. It is clear, for $c$ sufficiently small, that this will force $\Delta \gg c$ which simplifies the calculation. At the expense of exchanging an $m^{\prime}$ for an $m$, this gets us

$$
\Delta \geq m b^{\left[\frac{1+\frac{1}{\kappa}}{2+\frac{1}{q}}\right]}
$$

which, under our stipulation about $\kappa$, implies Equ.(3.65).

The rest of the argument is straightforward. Note that if we have the "weak excess of one" property for an interval of length $c^{\prime}$ then we also have it for an interval of length $c<c^{\prime}$. Suppose then that this happenstance occurs at some $t_{0}$, let us choose $c \ll 1$. Consider the situation at any time $t_{1}>t_{0}$. 
Dividing $\left[t_{0}, t_{1}\right]$ into small segments of size $b$ in accord with the derivation above, the backslide of the boundary will be of the order $\left(t_{1}-t_{0}\right) b^{\theta-1}$ i.e. if permitted, the boundary would be at $-\infty$ in zero time. However this is prevented by the boundary condition at $x=0$ and we may thus conclude that a density in excess of unity can never occur.

Proposition A.2. Consider the weak systems described under Hypotheses e-H.1 and t-H.2. Then, for $t \geq 0$, the boundaries are monotone and continuous. Moreover e.g. for the one-sided problem, under the slightly weaker condition that $\rho_{0}$ is not identically one, there is still monotonicity and, for $t>0$, continuity. Finally, for $t=0$ the boundary is continuous provided that $\rho_{0}$ is not identically 1 in a neighborhood of $L_{0}-$ with similar considerations on the right.

Proof. We again consider only the one sided problem with $0 \leq x \leq 1$ and note that $L \geq 0$. Without loss of generality, we may discuss only small times. Suppose there is a non-monotonicity; i.e. letting $t>0$ suppose that the set

$$
\mathscr{D}_{t}=\{s<t \mid L(s)>L(t)\}
$$

has positive measure. For any $s \in \mathscr{D}_{t}$ and let $G=\alpha(x)$ be another non-negative time independent convex function of the type used above: strictly positive on $[0, L(s))$ and vanishing for $x \geq L(s)$ along with all of its its derivatives. Placing this into Equ.(3.61), the right hand side is not negative and is strictly positive unless $\rho$ vanishes a.e. on the region $\{0 \leq x \leq L(t) ; s \leq \tau \leq t\}$. Meanwhile, the left hand side is equal to

$$
\int_{L(t)}^{L(s)}(\rho(x, t)-1) d x .
$$

which, using Proposition A.1, is non-positive and, unless $\rho(x, t)$ is a.e. equal to one between $L(t)$ and $L(s)$ is in fact strictly negative. So, assuming that a contradiction has not already been achieved, consider values $s^{\prime} \in(s, t)$. Either $L\left(s^{\prime}\right) \geq L(s)$ (a.e.) or, there is a set of positive measure where $L\left(s^{\prime}\right)<L(s)$. In the former case we immediately conclude that the time $t$ is "exceptional" and it may be removed. In the latter case, we run the same argument again leading to a primary contradiction or the conclusion that $\rho$ did not vanished a.e. on the set between $L(s)$ and $L(t)$ rendering a contradiction for the $s, t$ argument.

With the monotonicity in hand, we are in the realm where we may employ essentially classical arguments. Let us rule out the possibility of a discontinuity in $L(t)$ at time $t_{0}>0$. Let us define $\lim _{t \uparrow t_{0}} L(t)=L\left(t_{0}\right)$ and suppose that $\lim _{t \downarrow t_{0}} L(t)=L\left(t_{0}\right)+2 \Delta$. Note that for all $t$, all the boundary data - even at (or as we approach) the left - puts $\rho \leq 1$. Consider the density $\tilde{\rho}$ in $L\left(t_{0}\right)+\Delta \leq x \leq 1$ which has $\tilde{\rho}\left(L\left(t_{0}\right)+\Delta, t\right) \equiv 1$ and agrees with the boundary data for the current problem at $x=1$ and $t=0$. Now regardless of the situation for times $t>t_{0}$ when ostensibly the boundaries have crossed, for $t<t_{0}$, it is clear that on their common domain, $\rho(x, t) \leq \tilde{\rho}(x, t)$. Moreover, since $\tilde{\rho}$ is purely classical, it follows from the strong maximum principle that away from its boundaries, $\tilde{\rho}<1$. To finish the argument, let $G(x)$ be any positive test function supported in $\left[0, L\left(t_{0}\right)+2 \Delta\right]$; vanishing along with all of its derivatives at the endpoint. Then, using Equ.(3.61), and $t \lesssim t_{0}$, and an upper limit in very slight 
excess of $t_{0}$ we obtain

$$
\int_{L(t)}^{L\left(t_{0}\right)+2 \Delta}(1-\rho(x, t)) G d x=\int d x \int_{t}^{t_{0}} \rho(x, \tau) G_{x x} d \tau .
$$

The right hand side tends to zero as $t \uparrow t_{0}$ while on the left hand side, we are bounded below by

$$
\int_{L(t)+\Delta}^{L\left(t_{0}\right)+2 \Delta}(1-\rho(x, t)) G d x
$$

which is strictly positive.

A similar sort of argument holds at $t=0$ provided that $\rho_{0}$ is not identically one in a neighborhood of the starting point. (In the latter case, as we will show below, there is immediately, at $t=0$, some sort of a discontinuity.) All of the preceding arguments have assumed that times $t$ are small enough that we need not concern ourselves with collision at $x=K$ or between the two moving boundaries. By a straightforward resetting argument, all of the above can be achieved for any $t \leq T<t^{\star}$.

Remark 6. It seems clear that if $\rho_{0}$ is identically one in a neighborhood of the origin, then $L$ immediately jumps to the endpoint of the neighborhood; this can easily be established in the context of the particle system as will be discussed below. But, from the perspective of the continuum, these cases can be circumvented by redefining $\rho_{0}=0$ and $a_{0}=1$ in the neighborhood, i.e. it is just an inappropriate labeling of the resources. Notwithstanding, genuine discontinuities will occur in this system if the density gets bigger than one in the vicinity of the moving boundary - a circumstance that can develop dynamically. Indeed, we shall conclude this note with a lemma/example that indeed demonstrates the present problem to be borderline ill-posed.

Lemma A.3. If $\rho_{0}(x) \leq \rho_{F}$ with $\int \rho_{0}(x) d x=1+h>1$, then $L(t)$ disappears before it hits $x=1$. If $\rho_{0} \equiv \rho_{F}=1$, then $L(t)$ disappears instantly at $t=0$. Finally, if $\rho_{0} \equiv 1$ in $\left[0, x_{0}\right]$ but for $x>x_{0}$ satisfies $t$-H.2 with $L_{0}$ replaced by $x_{0}$ then there is a consistent solution with a discontinuity at $t=0$ and regular thereafter.

Proof. We start with the substantive portion: By plugging in smooth approximations of the step function $G(x)=\chi_{(-\infty, 1)}$ into the of weak equation, we obtain

$$
L(t)+\int \rho(x, t) d x=\int \rho_{0}(x) d x+\int_{0}^{t} \nabla \rho(1, s) d s,
$$

Starting with $L(0)=0$ we may assume that for a while $L(t)<1$ or there is nothing to prove. (Further, this guarantees that $\nabla \rho(1, s)$ makes sense for $0 \leq s \leq t$.) Since $\rho_{0}(x) \leq \rho_{F}$, by the maximum principle $\rho(x, t) \leq \rho_{F}$ and $\nabla \rho_{F} \geq 0$. Since $\rho(\cdot, t)$ is supported in $[L(t), 1]$, we obtain

$$
L(t)+(1-L(t)) \rho_{F} \geq \int_{0}^{1} \rho_{0}(x) d x=1+h .
$$

But if $L(t)$ gets sufficiently close to 1 , the quantity on the left hand side will be less than $1+h$. Hence we conclude that under the stated conditions, all assumptions of smoothness and continuity break 
down i.e. the boundary disappears at some positive time. If $\rho_{0} \equiv \rho_{F}=1$, then we would conclude $L+(1-L) \rho_{F}=1$ which, evidently, means that the boundary disappears instantly at $t=0$. As for the intermediate situation: It is seen by one last appeal to the weak equations, using arguments that are by now familiar, that $\lim _{t \downarrow 0} L(t)=x_{0}$ and, one the basis of everything else in this note, the solution for $t>0$ is identical to the one where the starting point is $x=x_{0}$. We shall omit the details.

Note that the primary case includes the situations where $\rho_{0}$ vanishes in a neighborhood of $x=0$; in these cases, the Stefan boundary is guaranteed to move smoothly for short times. Let us next discuss the final case which, from the perspective of the particle system, is not completely trivial. Indeed, suppose that the initial condition is such that in some small neighborhood to the right of $x_{0}$, the density vanishes. For the microscopic Stefan setup as described, there is a hole initially at $x=L_{0}=0$ whose dynamical location defines the moving boundary. Initially there is a wall of size $N x_{0}$. The time scale in lattice units that it will take the hole to get through this wall is of the order $N$. In the meanwhile, the right edge of the wall will have spread out - here to a distance of the order $N^{1 / 2}$. However, on this time scale, the particles to the right of the gap will only have closed a vanishing fraction of the gap. In macroscopic units, all of these events take place in times of the order $N^{-1}$. Thus, in the continuum limiting version, we would see precisely the discontinuity described in Lemma A.3.

\section{Acknowledgments}

This research was supported by the NSF under the grants DMS-07-00732 (IK) and DMS-03-06167 (LC).

\section{References}

[1] I. Athanasopoulos, L. Caffarelli and S. Salsa, Caloric Functions in Lipschitz Domains and the Regularity of Solutions to Phase Transition Problems, The Annals of Mathematics, 143 no. 3, 413-434 (1996)

[2] E. Di Benedetto and A. Friedman, The Ill-Posed Hele-Shaw Model and The Stefan Problem for Supercooled Water, Trans. Amer. Math. Soc. 282 no. 1, 183-204 (1984).

[3] L. Chayes and T.M. Liggett, One Dimensional Nearest Neighbor Exclusion Processes in Inhomogeneous and Random Environments, J. Statist. Phys. 129, 193-203 (2007)

[4] L. Chayes and G. Swindle, Hydrodynamic Limits for One-Dimensional Particle Systems with Moving Boundaries, Ann. Probab. 24, no.1, 559-598 (1996).

[5] L. Chayes, R.H. Schonmann and G. Swindle, Lifshitz' Law for the Volume of a Two-Dimensional Droplet at Zero Temperature, J. Statist. Phys. 79 821-831 (1995). 
[6] A. Fasano, and M. Primicerio, General Free-Boundary Problems for the Heat Equation, J. Math. Anal. Appl. 57 694-723 (1977).

[7] E. B. Fabes, Nicola Garofalo and Sandro Salsa Comparison Theorems for Temperatures in noncylindrical Domains, Atti Accad. Naz. Lincei, Read. Ser. 8. 78 1-12 (1984).

[8] A. Friedman, Variational Principles ad Free-Boundary Problems, Robert E. Krieger Publishing Company, INC. (1982)

[9] R.J. Glauber, Time Dependent Statistics of the Ising Model, J. Math. Phys. 4 294-307 (1963)

[10] S. W. Hawking Black Hole Explosions? Nature 248, 30-31 (1974)

[11] V. M. Isakov, Inverse theorems concerning the smoothness of potentials, Differential Equations11, 50-56 (1974), [translated from Russian].

[12] A. Karma and A.E. Lobkovsky Low-temperature dynamics of kinks on Ising interface, Phys. Rev. E 71036114 (2005).

[13] D. Kinderlehrer and L. Nirenberg. Regularity in free boundary value problems, Ann. Scu. Norm. Sup. Pisa 4 373-391 (1977).

[14] T. M.Liggett. Interacting Particle Systems, Springer Verlag: New York, Berlin, Heidelberg, Tokyo (1985).

[15] H. Rost, Nonequilibrium Behavior of a Many Particle Process: Density Profile and Local Equilibrium, Z. Wahrsch. Verw. Gebiete 58 41-54 (1981).

[16] H. Spohn, Interface Motion in Models with Stochastic Dynamics, J. Stat. Phys 71 1081-1132 (1993). 